\title{
Design, computational studies, synthesis and in vitro antimicrobial evaluation of benzimidazole based thio-oxadiazole and thio-thiadiazole analogues
}

Nada A. Noureldin ${ }^{1,2^{*}}$, Jennifer Richards ${ }^{3}$, Hend Kothayer ${ }^{2}$, Mohammed M. Baraka², Sobhy M. Eladl², Mandy Wootton ${ }^{3}$ and Claire Simons ${ }^{1}$

\begin{abstract}
Background: Two series of benzimidazole based thio-oxadiazole and thio-thiadiazole analogues were designed and synthesised as novel antimicrobial drugs through inhibition of phenylalanyl-tRNA synthetase (PheRS), which is a promising antimicrobial target. Compounds were designed to mimic the structural features of phenylalanyl adenylate (Phe-AMP) the PheRS natural substrate.

Methods: A 3D conformational alignment for the designed compounds and the PheRS natural substrate revealed a high level of conformational similarity, and a molecular docking study indicated the ability of the designed compounds to occupy both Phe-AMP binding pockets. A molecular dynamics (MD) simulation comparative study was performed to understand the binding interactions with PheRS from different bacterial microorganisms. The synthetic pathway of the designed compounds proceeded in five steps starting from benzimidazole. The fourteen synthesised compounds $\mathbf{5 a - d}, \mathbf{6 a - c}, \mathbf{8 a}-\mathbf{d}$ and $\mathbf{9 a - c}$ were purified, fully characterised and obtained in high yield.
\end{abstract}

Results: In vitro antimicrobial evaluation against five bacterial strains showed a moderate activity of compound $\mathbf{8 b}$ with MIC value of $32 \mu \mathrm{g} / \mathrm{mL}$ against S. aureus, while all the synthesised compounds showed weak activity against both E. faecalis and P. aeruginosa (MIC $128 \mu \mathrm{g} / \mathrm{mL}$ ).

Conclusion: Compound $\mathbf{8 b}$ provides a lead compound for further structural development to obtain high affinity PheRS inhibitors.

Keywords: Benzimidazole, Antimicrobial resistance, PheRS, Phe-AMP, Flexible alignment, Molecular dynamics

\section{Introduction}

Antimicrobial resistance is one of the most challenging medical dilemmas worldwide [1-4]. Antibacterial resistance annually leads to 700,000 deaths, and this number is estimated to reach 10 million annual deaths by 2050

\footnotetext{
*Correspondence: NANoureddine@pharmacy.zu.edu.eg

'School of Pharmacy and Pharmaceutical Sciences, Cardiff University, Cardiff CF10 3NB, UK

Full list of author information is available at the end of the article
}

[3]. The emergence of pan-drug resistant bacterial strains that are resistant to all known antimicrobial agents, have been reported [5], which is alarming and could result in a return to the 'post-antibiotic era' where a minor injury could be a life-threatening event [6]. Bacterial cells have exceptional survival genetic plasticity mechanisms that are highly flexible in encountering environmental threats [7], therefore efforts should be focused on decreasing antimicrobial resistance development. Improper and over prescription of broad-spectrum antibiotics are the 
main causes that exert a high pressure enhancing the natural genetic mutations in pathogenic bacteria [1]. Finding new targets within the bacterial cells is considered a promising strategy to overcome the high rates of antimicrobial resistance development.

Aminoacyl tRNA synthetases (aaRSs) are a group of enzymes that encode the aminoacylation of the tRNA in the protein synthesis process [8]. These enzymes are vital for the cell function and their inhibition leads to a cascade of events named 'the stringent response' resulting in cell growth cessation [9]. The availability of the resolved crystal structures of most of the aaRS enzymes together with their different structures in bacterial cells compared with their human counterparts, make them a hopeful antibacterial target [10-16].

The aaRS family consists of 23 enzymes, which are classified into two main classes (class I and class II) according to their active site structure and the enzyme kinetics [17]. Class I enzymes usually ligate the bulky amino acids [18], and the active site consists of two motifs: KMSKS (LysMet-Ser-Lys-Ser) and HIGH (His-Ile-Gly-His). Class I enzymes usually aminoacylate tRNA at the 2' hydroxy group of the ribose sugar at the tRNA CCA $3^{\prime}$ end [1822 . Class II aaRS enzymes are characterised by the presence of three active motifs in their active sites assigned motif I, II and III, and they have either dimeric or tetrameric structures. Class II members charge their cognate amino acids at the 3' hydroxy group of the A76 ribose at the $3^{\prime}$ end of the tRNA [18-22]. The tRNA aminoacylation reaction takes place in two main steps. Firstly, the amino acid is activated by interaction with ATP, and the product of this reaction is the highly reactive aminoacyl adenylate (aa-AMP) intermediate. Secondly, the esterification reaction between the carboxylic group of the amino acid and either the 2' or 3' hydroxy group of the terminal adenine ribose sugar of the $\mathrm{RNA}^{\text {aa }}$ occurs resulting in the aminoacyl charged tRNA (aa-tRNA ${ }^{\text {aa }}$ ) [18-22].

Among class II aaRS enzymes is the uniquely structured phenylalanyl-tRNA synthetase (PheRS). Bacterial PheRS is a heterodimer $(\alpha \beta)_{2}$ with two small $\alpha$ subunits (PheS) and two large $\beta$ subunits (PheT), and the aminoacylation active site is located in the $\alpha$ subunit. Although PheRS is a member of class II enzymes, it exclusively attaches phenylalanine at the 2' hydroxy group of its tRNA A76 ribose moiety $[18,19,21,23]$. The structure of the PheRS enzyme together with its active site interactions have been extensively studied [24-30]. The PheRS aminoacylation active site contains three main binding pockets: the phenylalanine, ATP and tRNA binding pockets, which are located close to each other in all aaRS enzymes [22]. Studies have revealed that the most active aaRS inhibitors are those that have a dual inhibition activity for two or more of these binding pockets as they exhibit the highest binding affinity [22].

This research describes the design and synthesis of PheRS inhibitors that have the ability to occupy both phenylalanine and ATP binding pockets at the same time. As the aminoacylation active site amino acid key residues are highly conserved among bacterial species, the design criteria should ensure the generation of broad-spectrum active antibiotics [9]. The compounds were designed to keep the same structural features of the Phe-AMP natural substrate to retain the same stabilising conserved interactions within the active aminoacylation pockets (Fig. 1). Based on this, two series of compounds; benzimidazole based aryl thio-oxadiazole analogues and benzimidazole based aryl thio-thiadiazole analogues were designed and examined by flexible alignment studies to ensure conformational similarity with the Phe-AMP natural substrate (Fig. 2). Furthermore, a molecular dynamics (MD) simulation comparative study was performed to understand the binding interactions of the designed compounds with PheRS from different bacterial microorganisms. The designed compounds were evaluated against a range of bacterial species to determine whether broad spectrum activity was obtained.

\section{Results and discussion \\ Computational studies}

The designed compounds were examined for their conformational similarity with the natural PheRS substrate Phe-AMP using the flexible alignment tool in Molecular Operating Environment (MOE) [31]. The results of some representative compounds from both series are shown in Fig. 3.

As revealed from the 3D flexible alignment results, the designed compounds have similar conformations to the natural substrate Phe-AMP. The benzimidazole moiety in the designed scaffold overlaps with the adenine moiety of Phe-AMP, and both the oxadiazole and thiadiazole moieties overlap with the phosphate group of the natural substrate, while the terminal aromatic moiety aligns with the phenylalanine aromatic ring. As the complementary moieties share nearly the same chemical features, and the designed compounds share the same 3D conformation of the natural substrate, it was expected that they would share the same active site interactions.

A docking study using Thermus thermophilus PheRS [32] was performed (Fig. 4), Th. thermophilus was chosen for this study as it is the only resolved crystal structure with the complete enzyme substrate (Phe-AMP), while other available crystal structures were resolved with either unbound substrate or with inhibitors. Also, a multiple sequence alignment using PheRS amino acid sequences from five different bacterial species 

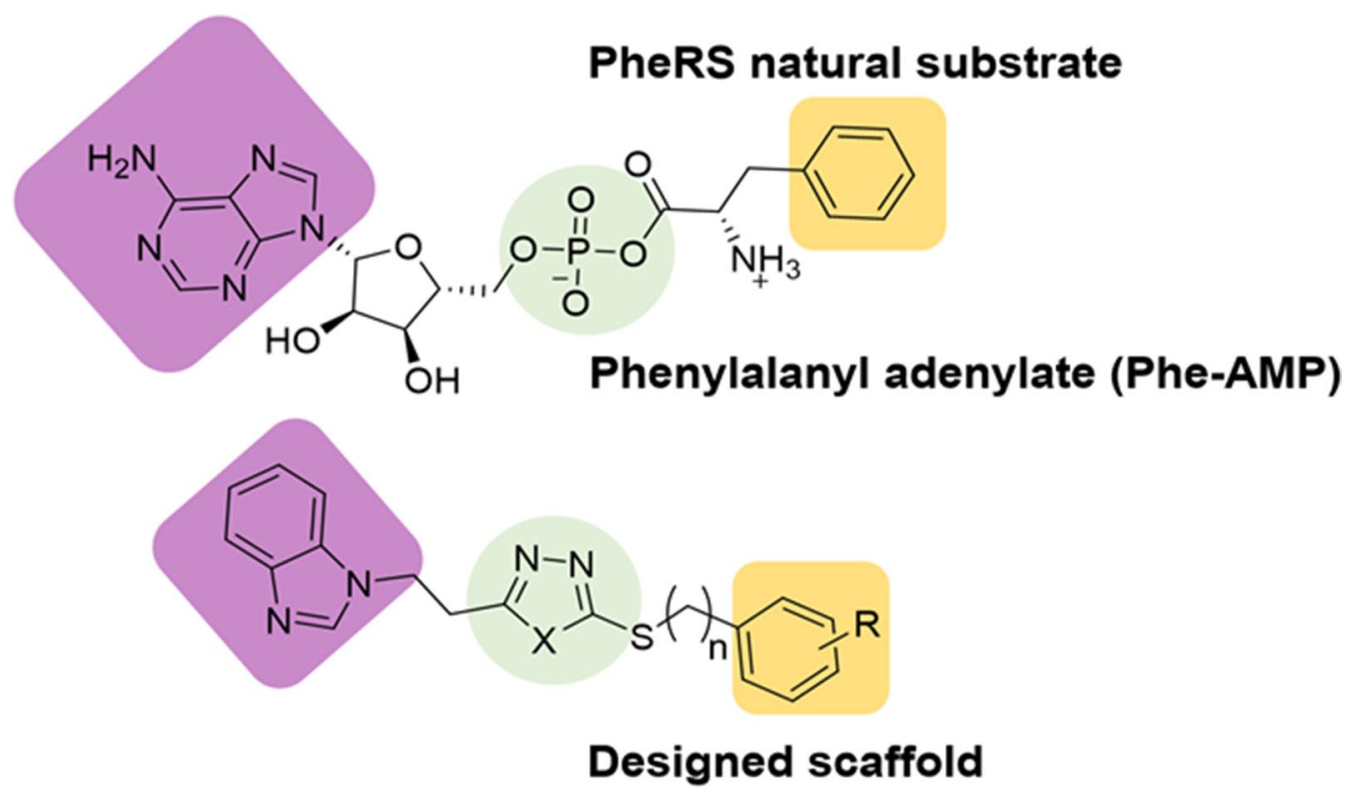

Fig. 1 The main features of Phe-AMP and the corresponding structure of the designed compounds

was performed to ensure the high level of conservation between the aminoacylation active site key amino acid residues among bacterial strains (Fig. 5). The two series of compounds were designed to occupy the Phe-AMP binding pockets in the PheRS aminoacylation active site, and to have the same binding interactions of the natural substrate. Benzimidazole was used as the base for synthesis to mimic the adenine moiety of Phe-AMP and to stabilise the compound in the active site pocket by hydrophobic interactions and hydrogen bonding with the conserved phenylalanine and arginine residues in this position, respectively (Phe216 and Arg321 in Th. thermophilus PheRS). Benzimidazole was chosen rather than the more hydrophilic adenine to improve the overall hydrophobicity of the compounds with the aim of improving permeability. Either oxadiazole or thiadiazole rings were positioned to allow the hydrophilic interactions at the ATP binding pocket comparable with the phosphate group in Phe-AMP (Arg204 in Th. thermophilus PheRS). While the phenyl ring (free or substituted) was positioned to resemble the phenyl ring of the phenylalanine in Phe-AMP. The presence of an aryl group at this position is important to stabilise the structure in the Phe-AMP binding pocket with $\pi$ stacking with the conserved phenylalanine residues in that position of the active site (Phe258 and Phe260 in Th. thermophilus PheRS). Docking studies suggested that the designed compounds can occupy the aminoacylation active site encountering the key amino acid residues responsible for the natural substrate binding (Fig. 4). Due to the high level of conservation between the PheRS aminoacylation active sites among bacteria as revealed from the multiple sequence alignment, the aim was to design broad-spectrum antimicrobial agents sharing the same binding criteria as the substrate (Fig. 5).

Molecular dynamics was performed for compounds 5c, $\mathbf{6 a}, \mathbf{8 b}$ and $9 \mathbf{b}$. Each of the compounds chosen represents those with the best docking binding interaction in each series. The PheRS-ligand complexes were subjected to a 200 ns simulation (Fig. 6). The final frame of compound $\mathbf{5 c}$ showed the stabilisation of the compound in the phenylalanine binding pocket by hydrophobic interaction between the para-fluorophenyl group and the key amino acid residue Phe260, and the stabilisation of the compound in the binding pocket by hydrophilic interaction between the sulphide group and Gly316, while the benzimidazole moiety of the structure moved away from the adenine binding pocket. It is important to mention that the protein- ligand $\mathbf{5 c}$ complex did not reach equilibrium during the simulation time. The final frame of compound 6a showed the best fit in all binding pockets with the structure stabilised in the phenylalanine binding pocket via $\pi$ stacking with the key amino residues Phe258 and Phe260. Compound 6a was also stabilised in the phosphate binding pocket by hydrogen bonding between the sulphide group and Gly316 and stabilised in the ribose binding pocket by hydrophobic interaction with Arg204 and water mediated interaction with Met148, while being stabilised in the adenine binding pocket by hydrophobic interaction with Phe216. However, the final frame 
<smiles>Nc1ncnc2c1ncn2[C@@H]1O[C@H](COP(=O)([O-])OC(=O)[C@H]([NH3+])Cc2ccccc2)[C@@H](O)[C@H]1O</smiles>

Phenylalanyl adenylate (Phe-AMP)<smiles>[R]c1ccc(CSc2nnc(CCn3cnc4ccccc43)o2)cc1</smiles>

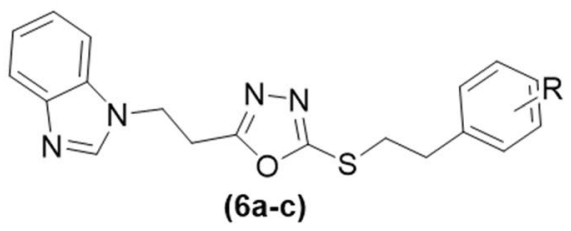

Benzimidazole based aryl thio-oxadiazole analogues Series 1

\begin{tabular}{l|l}
$\mathbf{R}$ & COMPOUND \\
\hline $\mathrm{H}$ & $\mathbf{5 a}$ \\
$2,4-$ dichloro & $\mathbf{5 b}$ \\
$4-\mathrm{F}$ & $\mathbf{5 c}$ \\
$4-\mathrm{C}=\mathrm{N}$ & $\mathbf{5 d}$ \\
$\mathrm{H}$ & $6 \mathbf{a}$ \\
$4-\mathrm{Cl}$ & $6 \mathbf{b}$ \\
$4-\mathrm{OCH}_{3}$ & $6 \mathrm{c}$
\end{tabular}<smiles>[R]1cccc(CSc2nnc(CCn3cnc4ccccc43)s2)c1</smiles><smiles></smiles>

Benzimidazole based aryl thio-thiadiazole analogues Series 2

\begin{tabular}{l|l}
$\mathbf{R}$ & COMPOUND \\
\hline $\mathrm{H}$ & $\mathbf{8 a}$ \\
$2,4-$ dichloro & $\mathbf{8 b}$ \\
$4-\mathrm{F}$ & $\mathbf{8 c}$ \\
$4-\mathrm{C} \equiv \mathrm{N}$ & $\mathbf{8 d}$ \\
$\mathrm{H}$ & $9 \mathrm{a}$ \\
$4-\mathrm{Cl}$ & $\mathbf{9 b}$ \\
$4-\mathrm{OCH}_{3}$ & $\mathbf{9 c}$
\end{tabular}

Fig. 2 The structure of phenylalanyl adenylate (Phe-AMP) and the structures of series 1 and 2 designed compounds

interactions obtained for compounds $\mathbf{8 b}$ and $\mathbf{9 b}$ were unexpected. Both compounds protruded outside the binding pockets while remaining connected to the active site via hydrophobic interaction with Phe 258 that were maintained for more than $60 \%$ of the simulation time for the two compounds. From these computational data it was expected that series 1 compounds would show better activity than series 2 compounds.

Unexpectedly, the microbiological evaluation using S. aureus, E. faecalis, $P$. aeruginosa, $K$. pneumoniae and $E$. coli revealed that compound $\mathbf{8 b}$ from series 2 had the best activity against $S$. aureus (MIC $32 \mu \mathrm{g} / \mathrm{mL}$ ), however, it had poor molecular interactions with Th. thermophilus PheRS. All the compounds from the two series showed weak inhibitory activity against $E$. faecalis and $P$. aeruginosa (MIC $128 \mu \mathrm{g} / \mathrm{mL}$ ) and were inactive against both E. coli and $K$. pneumoniae. To provide a clearer insight of these unexpected results on a molecular level a published homology model of S. aureus PheRS [34] was used to study the interactions between $\mathbf{8 b}$ and the S. aureus PheRS enzyme using MD simulation. On visualising the final frame 2D interactions of the simulation using MOE software (Fig. 7) hydrophobic interactions with both the conserved Phe212 and the conserved Phe254 stabilising the structure in the right pocket were observed. Moreover, hydrogen bonding between N3 in the thiadiazole ring and the conserved Arg318 (Arg321 in Th. thermophilus PheRS) and between N3 in the benzimidazole ring and Ser209 were observed. On monitoring the MD protein ligand diagram (Fig. 8) it shows strong 


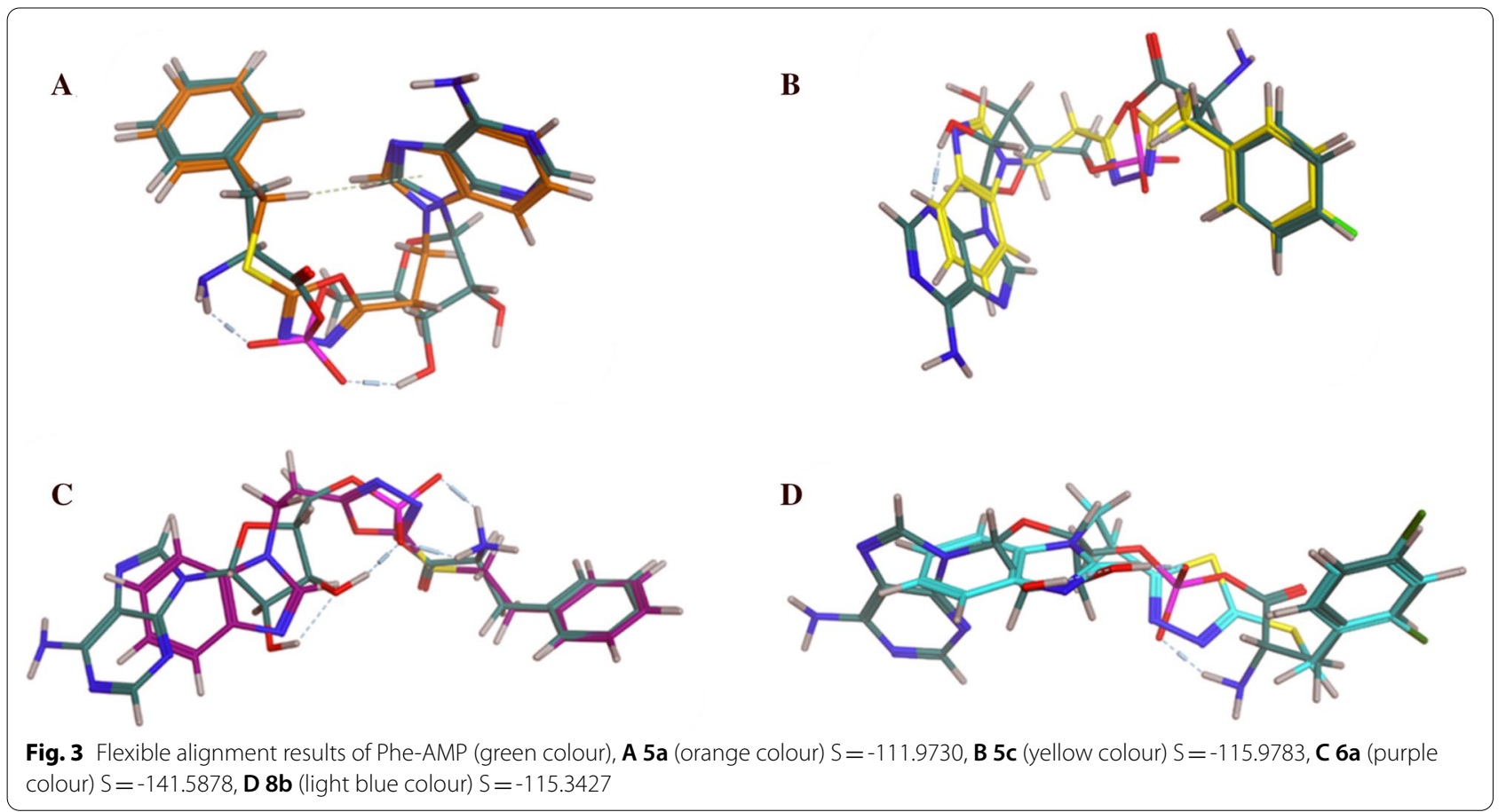

hydrophobic stabilisation of the compound inside the right pocket throughout the simulation time with both Phe212 (interaction maintained more than $80 \%$ of the simulation time) and Phe254 (interaction maintained for more than $60 \%$ of the simulation time). A strong hydrogen bond was maintained for about $80 \%$ of the simulation time with Ser209 and fair hydrophilic interactions with Arg318 were maintained for more than $20 \%$ of the simulation time. The strong hydrogen bonding with Ser209 seems to be highly important to hold the structure in the ATP binding site, stabilising the structure in the intended conformation contributing to the more promising activity. This agrees with the results revealed in a study by Wang et al. [29], which found that good interactions in the ATP binding site offers a great opportunity to inhibit both phenylalanine and ATP binding pockets resulting in a bi-substrate promising inhibitor.

The binding free energy $(\Delta G)$ for compound $\mathbf{8 b}$ complexed with the $S$. aureus homology model was calculated from each frame starting from the last $100 \mathrm{~ns}$ with respect to the RMSD (Fig. 9). The calculated $\Delta G$ of $-69.0431 \pm 3.23 \mathrm{kcal} / \mathrm{mol}$ indicated an optimal positioning within the active site and a high ligand binding affinity.

These results highlighted that despite the active site residues conservation in various bacterial PheRSs, there are distinct differences between inhibitor interactions, as illustrated by the MD interactions of $\mathbf{8 b}$, which were completely different against both $S$. aureus and
Th. thermophilus PheRSs (Fig. 10). This difference in ligand-protein interactions would make it more difficult to obtain a broad-spectrum antimicrobial agent from such a design strategy. Despite this, compound $\mathbf{8 b}$ (benzimidazole based thio-thiadiazole) was identified as a bi-substrate lead compound for further structural development to obtain high affinity $S$. aureus PheRS inhibitors. Future design strategies of PheRS inhibitors should focus on maintaining strong binding interactions in the ATP binding pocket to ensure correct positioning of the designed compounds in the Phe-AMP binding sites to achieve both high inhibitory activity and low resistance opportunity.

\section{Chemistry}

Compounds 5a-d, 6a-c, 8a-d and 9a-c were then synthesised following Fig. 11.

Methyl 3-(1H-benzo[d]imidazol-1-yl)propanoate (2) was synthesised by a nucleophilic substitution reaction between benzimidazole (1) and methyl 3-bromopropionate in the presence of $\mathrm{K}_{2} \mathrm{CO}_{3}$ and 18-crown-6, which is used to enhance the ionisation of $\mathrm{K}_{2} \mathrm{CO}_{3}$ in DMF [35-37]. 3-(1H-Benzo[d]imidazol-1-yl)propane hydrazide (3) was synthesised by hydrazinolysis of methyl 3-(1H-benzo[d]imidazol-1-yl)propanoate (2) using excess hydrazine hydrate at room temperature in $\mathrm{EtOH}[38,39]$. The hydrazide intermediate was used to synthesise two more intermediates, (4) and (7), following the reported procedure [40]. The hydrazide 

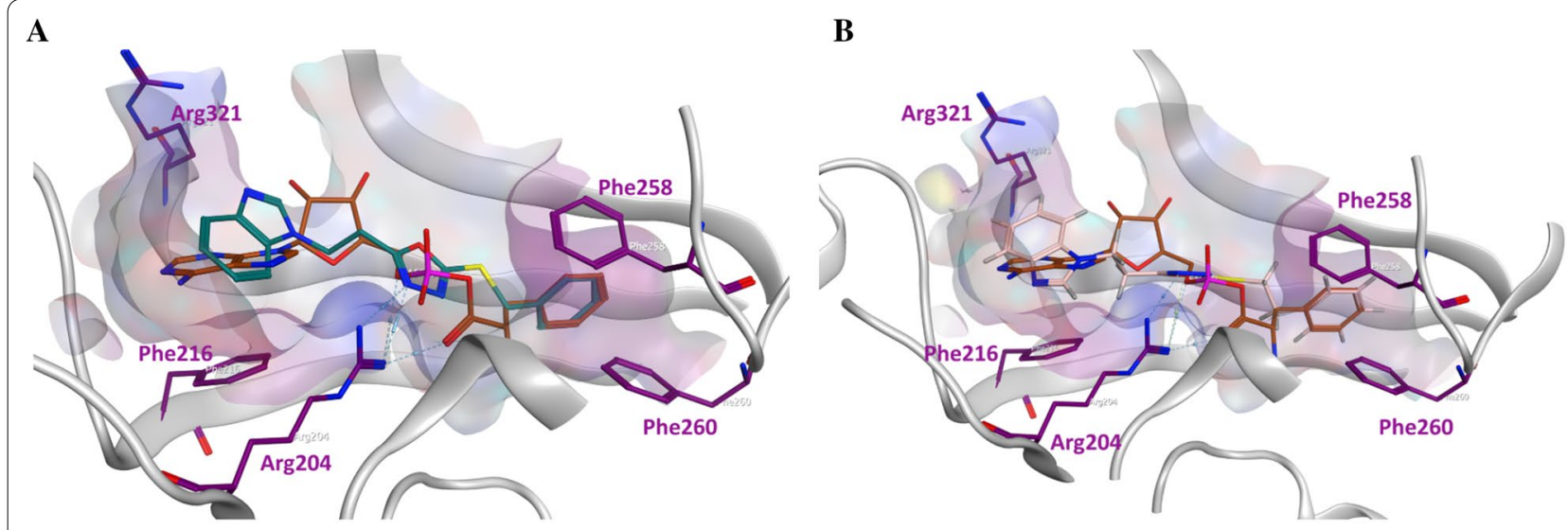

C

D
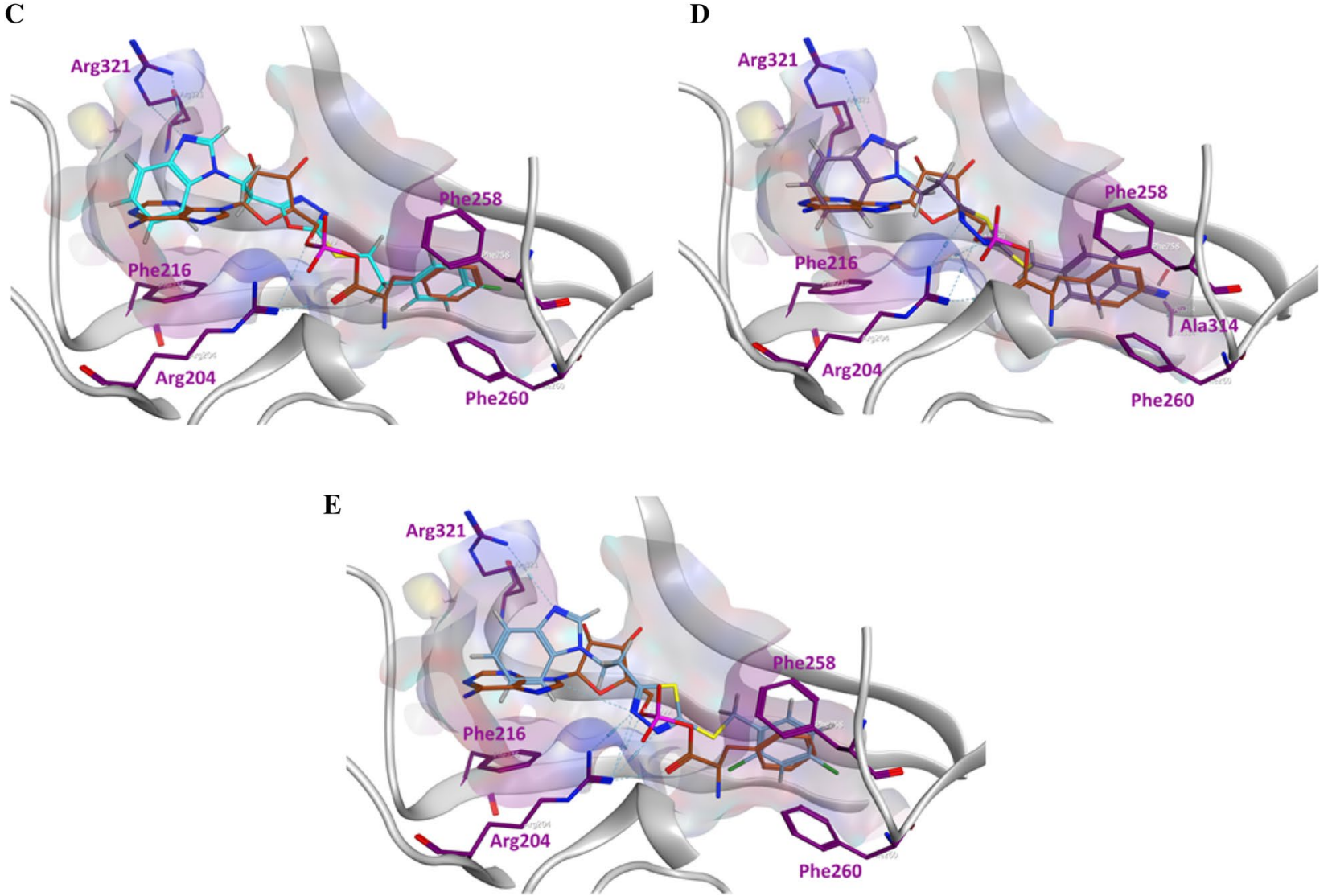

Fig. 4 Docking results of some of the designed compounds in the aminoacylation active site of Th. thermophilus PheRS [pdb: 1JJC] [32]. The Phe-AMP (orange colour) was placed to compare the binding mode of the natural substrate and the designed compound. The illustrated designed compounds are $\mathbf{A} \mathbf{5 a}$ (green colour), B 6a (pink colour), C $\mathbf{6 b}$ (light blue colour), D 8d (violet colour) and E $\mathbf{8 b}$ (blue colour). Some key residues in the active site are shown: Phe216 stabilises the adenine of Phe-AMP in the ATP binding site, Arg204 and Arg312 are responsible for hydrophilic interactions of AMP in the ATP binding pocket, and Phe258 and Phe260 stabilise the phenyl ring of the phenylalanine in the binding pocket with $\pi-\pi$ stacking

was first refluxed with $\mathrm{KOH}$ and carbon disulphide to afford 2-(3-(1H-benzo[d]imidazol-1-yl)propanoyl) hydrazine-1-carbodithioate intermediate, which was cyclised either into 5-[2-(1H-benzo[ $d]$ imidazol-1-yl) ethyl)-1,3,4-oxadiazole-2-thiol (4) by acidification with $1 \mathrm{~N}$ aqueous $\mathrm{HCl}$ or into $5-(2-(1 H$-benzo $[d]$ imidazol-1-yl)ethyl]-1,3,4-thiadiazole-2-thiol (7) by acidification with conc. $\mathrm{H}_{2} \mathrm{SO}_{4}$, with structure confirmation 
by high resolution mass spectroscopy. Reaction of 5-[2-(1H-benzo[d]imidazol-1-yl)ethyl]-1,3,4-oxadiazole-2-thiol (4) and 5-[2-(1H-benzo[d]imidazol-1-yl) ethyl]-1,3,4-thiadiazole-2-thiol (7) with various alkyl halides in the presence of $\mathrm{K}_{2} \mathrm{CO}_{3}$ afforded the target compounds 5a-d, 6a-c, 8a-d and 9a-c (Table 1).

\section{Microbiological evaluation}

All series 1 and 2 compounds were tested against $S$. aureus, E. faecalis, P. aeruginosa, E. coli and K. pneumoniae using ciprofloxacin as a reference drug. Microbiological evaluation indicated a moderate activity of compound $\mathbf{8 b}$ against S. aureus (MIC $32 \mu \mathrm{g} / \mathrm{mL}$ ). The structurally similar compound $\mathbf{5 b}$ showed weak inhibitory activity against S. aureus (MIC $128 \mu \mathrm{g} / \mathrm{mL}$ ), while all other compounds had poor activity against $S$. aureus (MIC $>128 \mu \mathrm{g} / \mathrm{mL}$ ). All the compounds showed weak inhibitory activity against both $E$. faecalis and $P$. aeruginosa (MIC $128 \mu \mathrm{g} / \mathrm{mL}$ ), and all the compounds were inactive against both $E$. coli and $K$. pneumoniae (MIC $>128 \mu \mathrm{g} / \mathrm{mL}$ ) except for compound 6c, which had weak inhibitory activity against $K$. pneumoniae (MIC $128 \mu \mathrm{g} / \mathrm{mL}$ ).

\section{Conclusion}

Development of PheRS inhibitors has been a promising strategy with good antimicrobial results $[29,30,41-$ 45]. In particular, drugs that have a dual inhibition of both phenylalanine and ATP binding pockets have the highest binding affinity [22]. Two series of compounds were designed to occupy both phenylalanine and ATP binding pockets, and to mimic Phe-AMP to retain the main stabilising binding interactions of the natural substrate. A comparative computational study revealed that, although the PheRS amino acid sequence is highly conserved among bacterial species, their interactions with enzyme inhibitors are completely different. The reasons behind such findings need more structural and crystallographic studies to optimise the rational design of inhibitors. Compounds were synthesised and evaluated for their in vitro antimicrobial activity against five bacterial strains (Gram + ve and Gram -ve). Only compound $\mathbf{8 b}$ showed a moderate activity against $S$. aureus with MIC of $32 \mu \mathrm{g} / \mathrm{mL}$.

\section{Experimental \\ Chemistry \\ General}

All reagents and solvents employed were of general purpose or analytical grade and purchased from Fluka, Acros, Alfa-Aeser and Sigma-Aldrich. Solvents were appropriately dried over molecular sieves $(4 \AA)$. ${ }^{1} \mathrm{H}$ and ${ }^{13} \mathrm{C}$ NMR spectra were recorded on a Bruker Advance DP500 spectrometer operating at $500 \mathrm{MHz}$ and $125 \mathrm{MHz}$ respectively. Chemical shifts are given in parts per million (ppm) relative to the internal standard tetramethylsilane $\left(\mathrm{Me}_{4} \mathrm{Si}\right)$. Coupling constants ( $J$ value) were calculated in hertz $(\mathrm{Hz})$. An additional file shows these spectra in more details (see Additional file 1). Silica gel Fluka Kieselgel 60, particle size 35-70 $\mu \mathrm{m}$ Davisil $^{\circledR}$ chromatography grade, was used for column chromatography in a glass column. Gradient column chromatography was performed with the aid of a pump. Analytical thin layer chromatography (TLC) was carried out on precoated silica plates (ALUGRAM ${ }^{\circledR}$ SIL G/UV254) with visualisation via UV light $(254 \mathrm{~nm})$. Melting points were determined using a Gallenkamp melting point apparatus and are uncorrected. UV HPLC was performed at the University of Bath using a sheath gas temperature of $350{ }^{\circ} \mathrm{C}$, flow rate of $12 \mathrm{~L} / \mathrm{min}$, and nebuliser gas at $45 \mathrm{psi}$ (3.10 bar). MS was calibrated using reference calibrant introduced from the independent ESI reference sprayer. Chromatographic separation was performed on a Zorbax Eclipse Plus C18 Rapid Resolution column $2.1 \times 50 \mathrm{~mm}, 1.8 \mu \mathrm{m}$ particle size using $\mathrm{H}_{2} \mathrm{O}$ (Merck, LC-MS grade) with $0.1 \%$ formic acid (FA, Fluka) v/v and methanol (MeOH, VWR, HiPerSolv) with $0.1 \%$ FA v/v as mobile phase $\mathrm{A}$ and $\mathrm{B}$, respectively.

Compounds 2 and 3 were prepared as previously described $[35-39,46]$.

\section{5- [2-(1H-benzo[d]imidazol-1-yl) ethyl]-1,3,4-oxadiazole-2-thiol (4)}

A mixture of 3-(1H-benzo[d]imidazol-1-yl)propane hydrazide $(3)(1 \mathrm{~g}, 4.9 \mathrm{mmol})$ and $\mathrm{KOH}(0.27 \mathrm{~g}$, $4.9 \mathrm{mmol})$ in absolute EtOH $(12 \mathrm{~mL})$ was allowed to stir at room temperature for $30 \mathrm{~min}$, then $\mathrm{CS}_{2}(0.56 \mathrm{~g}$, $7.35 \mathrm{mmol}$ ) was added and the reaction mixture was heated under reflux overnight. The solvent was removed under vacuum and $\mathrm{H}_{2} \mathrm{O}(20 \mathrm{~mL})$ was added and the resulting solution was cooled and acidified with $1 \mathrm{~N}$ aqueous $\mathrm{HCl}$. The formed solid was collected by filtration, washed with $\mathrm{H}_{2} \mathrm{O}$ and dried under vacuum at

(See figure on next page.)

Fig. 5 Multiple sequence alignment [33] of PheRS a subunit amino acid sequences from Th. thermophilus (Q5SGX2), P. aeruginosa (Q9I0A3), K. pneumoniae (A6TAI3), E. coli (P08312), S. aureus (P68849) and E. faecalis (Q836J6). The purple box shows the conserved phenylalanine residue responsible for hydrophobic stabilisation of the adenine moiety in Phe-AMP. The green boxes show the conserved arginine residues responsible for the hydrophilic interactions that stabilise the AMP moiety of the natural substrate. The yellow boxes show the conserved phenylalanine residues responsible for positioning of the phenyl ring of the Phe-AMP by face-to-edge hydrophobic interactions 
CLUSTAL O(1.2.4) multiple sequence alignment

Sp $\mid$ Q5SGX2|SYFA_THET8 SP|Q9I0A3 |SYFA_PSEAE SP|A6TAI3 $\mid$ SYFA KLEP7 SP|P08312|SYFA_ECOLI sp|P68849|SYFA_STAAU Sp|Q836J6|SYFA_ENTFA

SP|Q5SGX2|SYFA THET8 SP|Q9IOA3 |SYFA_PSEAE SP|A6TAI3|SYFA_KLEP7 SP|P08312|SYFA_ECOLI sp|P68849|SYFA_STAAU sp|Q836J6|SYFA_ENTFA

sp|Q5SGX2|SYFA_THET8 SP|Q9I0A3|SYFA_PSEAE SP|A6TAI3|SYFA_KLEP7 sp|P08312|SYFA_ECOLI Sp|P68849|SYFA_STAAU sp|Q836J6|SYFA_ENTFA

sp|Q5SGX2|SYFA_THET8 sp|Q9I0A3|SYFA_PSEAE Sp|A6TAI3 |SYFA KLEP7 sp|P08312|SYFA_ECOLI sp|P68849|SYFA_STAAU sp|Q836J6|SYFA_ENTFA

SP|Q5SGX2|SYFA_THET8 SP|Q9IOA3 |SYFA_PSEAE sp|A6TAI3|SYFA_KLEP7 Sp|P08312|SYFA_ECOLI sp|P68849|SYFA_STAAU sp|Q836J6|SYFA_ENTFA

sp|Q5SGX2|SYFA_THET8 sp|Q9IOA3|SYFA_PSEAE sp|A6TAI3|SYFA_KLEP7 sp|P08312|SYFA_ECOLI Sp|P68849|SYFA_STAAU sp|Q836J6|SYFA_ENTFA

Sp|Q5SGX2|SYFA_THET8 sp|Q9I0A3|SYFA_PSEAE SP|A6TAI3|SYFA_KLEP7 sp|P08312|SYFA_ECOLI sp|P68849|SYFA_STAAU sp|Q836J6|SYFA_ENTFA
- - - - MLEEALAAIQNARDLEELKALKARYLGKKGLLTQEMKGLSALPLEERRKRG - - MENLDALVSQALEAVRHTEDVNALEQIRVHYLGKKGELTQVMKTLGDLPAEERPKVG - - MSHLAELVASAKAAINEASDVAALDNVRVEYLGKKGHLTLQMTTLRELPPEERPAAG - - MSHLAELVASAKAAISQASDVAALDNVRVEYLGKKGHLTLQMTTLRELPPEERPAAG MSEQQTMSELKQQALVDINEANDERALQEVKVKYLGKKGSVSGLMKLMKDLPNEEKPAFG MTLQAQLEALRDNTLKEIAQVATLKELNQIRVETLGKKGPITEVLRGMKNLSPEERPVVG

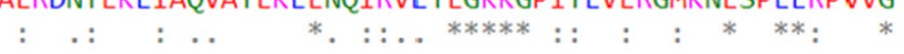

QELNAIKAALEAALEAREKALEEAALKEALERERVDVSLPGASLFSGGLHPITLMERELV 111 ALINVAKEKVQDVLNARKTELEGAALAARLAAERIDVTLPGRGQLSGGLHPVTRTLERIE 117 AVINEAKEQVQQALNARKAELEGAALNARLAAETIDVSLPGRRIENGGLHPVTRTIDRIE 117 AVINEAKEQVQQALNARKAELESAALNARLAAETIDVSLPGRRIENGGLHPVTRTIDRIE 117 QKVNELRQTIQNELDERQQMLVKEKLNKQLAEETIDVSLPGRHIEIGSKHPLTRTIEEIE 120 GFANEIRDLLTEAIEARKVVLENEALNAALKEESLDVTLPGKQMPQGTRHILTQVMEEIE 120

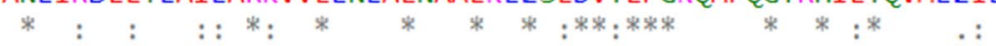

EIFRALGYQAVEGPEVESEFFNFDALNIPEHHPARDMWDTFWLTGEGFRLEGPLGEEVEG 171 QCFSRIGYEVAEGPEVEDDYHNFEALNIPGHHPARAMHDTFYFNA . . . . . . . . . 162 SFFGELGFTVATGPEIEDDYHNFDALNIPGHHPARADHDTFWFDA _.......... 162 SFFGELGFTVATGPEIEDDYHNFDALNIPGHHPARADHDTFWFDT _... . . . . . 162

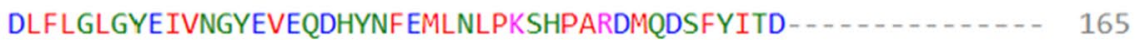

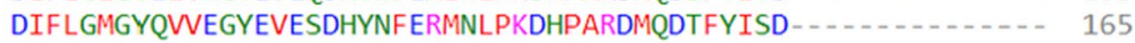
. $*$ : : . **:*. :..**: :*:* **** *:*:;

RLLLRTHTSPMQVRYMVAH- - TPPFRIVVPGRVFRFEQTDATHEAVFHQLEGLVVGEGI 228 NMLLRTHTSPVQVRTMESQ---QPPIRIVCPGRVYRCDSD-LTHSPMFHQVEGLLVDEGV 218 TRLLRTQTSGVQIRTMENQ---QPPIRIIAPGRVYRNDYD-QTHTPMFHQMEGLIVDKNI 218 TRLLRTQTSGVQIRTMKAQ- - -QPPIRIIAPGRVYRNDYD-QTHTPMFHQMEGLIVDTNI 218 EILLRTHTSPVQARTMESR-HGQGPVKIICPGKVYRRDSDDATHSHQFTQIEGLVVDKNV 224 EMLIRTHTSPVQARTMEKHDFSKGALRMISPGKVFRRDTDDATHSHQFHQIEGLVVDKNV 225 $*: * *: * *: * * * \quad: \quad .::: * *: *: *: \quad * * \quad * *: * * *: *$. :

AMAHLKGAIYELAQALFGPDSKVRFQPVYFPFVEPGAQFAVWW-.......PEGGKWL 278 SFADLKGTIEEFLRAFFEKQLEVRFRPSFFPFTEPSAEVDIQCVICSGNGCRVCKQTGWL 278 SFTNLKGTLHDFLNNFFEEDLQVRFRPSYFPFTEPSAEVDVM_.....-GKNGKWL 267 SFTNLKGTLHDFLRNFFEEDLQIRFRPSYFPFTEPSAEVDVM -........-GKNGKWL 267 KMSDLKGTLELLAKKLFGADREIRLRPSYFPFTEPSVEVDVSCFKCKGKGCNVCKHTGWI 284 TMGDLKGTLEVMMKKMFGEDRKIRLRPSYFPFTEPSVEVDVSCFKCGGAGCNVCKHTGWI 285

$$
: . * * *:: \text { : . : : }:::^{*}::^{*}: * * * * * \ldots: \text { : : }: \text { : }
$$

ELGGAGMVHPKVFQAVDAYRERLGLPPAYRGVTGFAFGLGVERLAMLRYGIPDIRYFFGG 338 EVMGCGMVHPNVLRMSNIDP - ...... EKFQGFAFGMGAERLAMLRYGVNDLRLFFDN 329 EVLGCGMVHPNVLRNVGIDP _........ EVYSGFAFGMGMERLTMLRYGVTDLRAFFEN 318 EVLGCGMVHPNVLRNVGIDP - . - . - EVYSGFAFGMGMERLTMLRYGVTDLRSFFEN 318 EILGAGMVHPNVLEMAGFDS - . - . - . SEYSGFAFGMGPDR IAMLKYGIEDIRHFYTN 335 EILGAGMVHPDVLQMSGIDP ........ - TEYSGFAFGLGPDRVAMLRYGVNDIRNFYQN 336 $*$ : *.*****.*: . *****:* :*: :**:**: *:**:.

RLKFLEQFKGVL_-..- 350

DLRFLGQFR - .... 338

DLRFLKQFK_..... 327

DLRFLKQFK_..... 327

DVRFLDQFKAVEDRGDM 352

DLRFLNQFKVKE - - - 348 $:$ : ****:

Fig. 5 (See legend on previous page.) 


\section{Protein-Ligand Contacts}

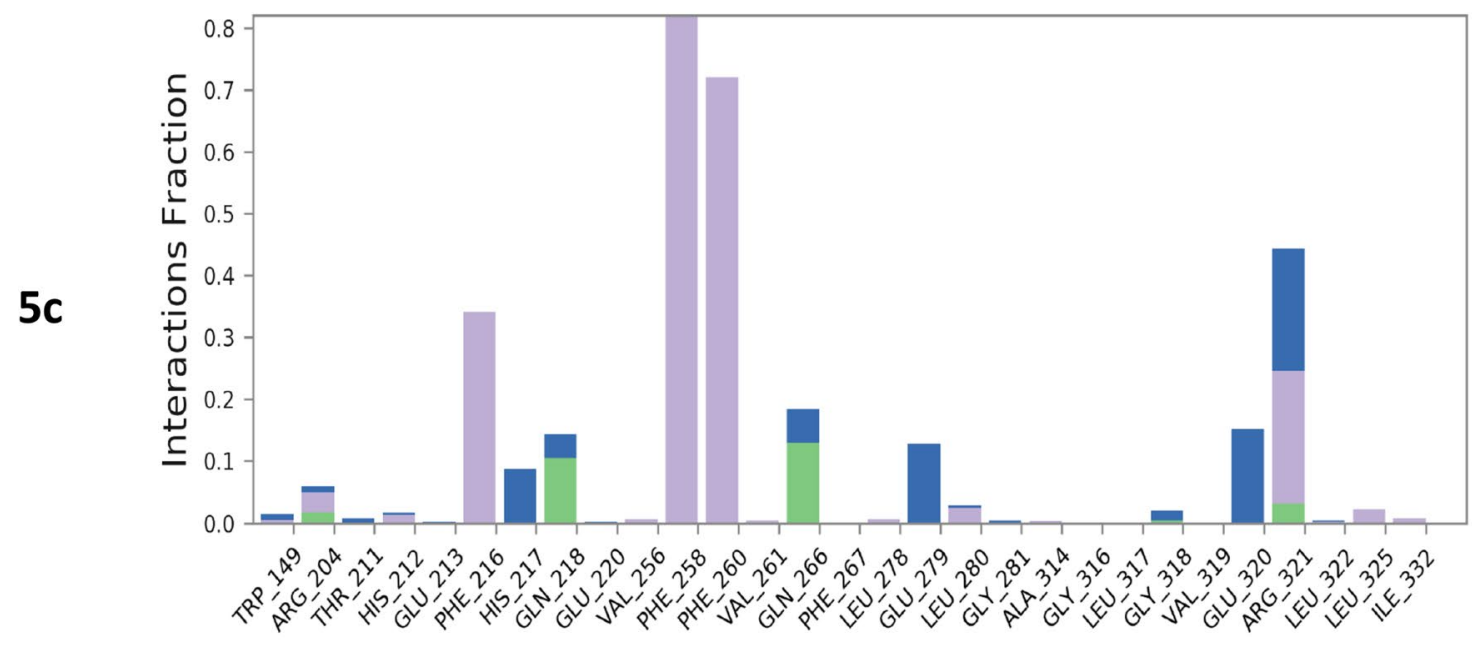

H-bonds Hydrophobic $\square$ lonic $\square$ Water bridges

\section{Protein-Ligand Contacts}

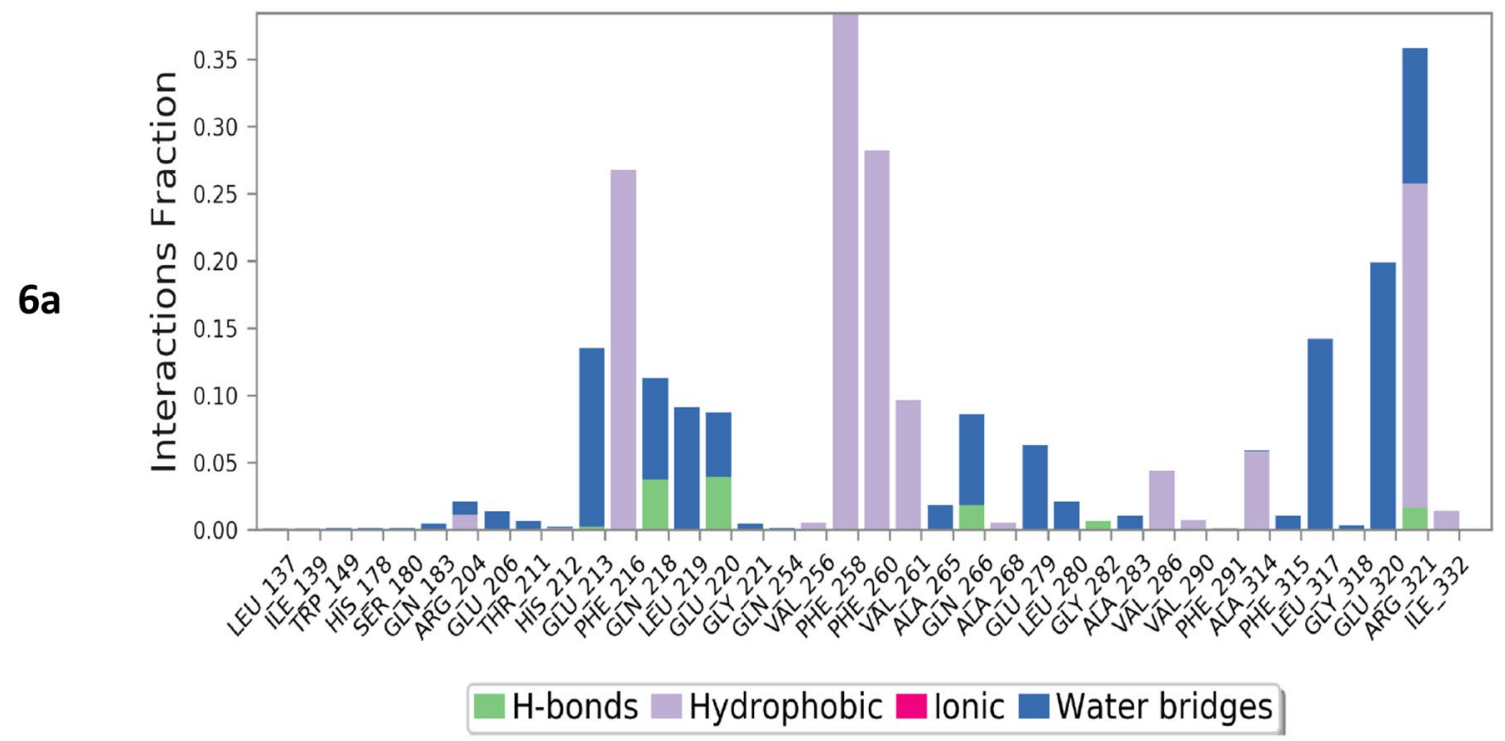

Fig. 6 Simulation interaction diagrams showing the protein-ligand interactions with Th. thermophilus PheRS throughout the $200 \mathrm{~ns}$ simulation. The stacked bar charts are normalised over the course of the trajectory, for example a value of 0.4 indicates that the specific reaction is maintained $40 \%$ of the simulation time

$40{ }^{\circ} \mathrm{C}$ to obtain the product as a light brown solid: Yield: 1.11 g $(92 \%)$; m.p. $225-227{ }^{\circ} \mathrm{C}$; HPLC: $95.65 \%$ at R.T. $3.27 \mathrm{~min} ;{ }^{1} \mathrm{H}$ NMR (DMSO- $\left.d_{6}\right) \delta: 3.33(\mathrm{t}, J=6.8 \mathrm{~Hz}$, $\left.2 \mathrm{H}, \mathrm{NCH}_{2} \mathrm{CH}_{2}\right), 4.65\left(\mathrm{t}, J=6.8 \mathrm{~Hz}, 2 \mathrm{H}, \mathrm{NCH}_{2}\right), 7.22$ (td, $J=1.2,7.2 \mathrm{~Hz}, 1 \mathrm{H}, \mathrm{Ar}), 7.28(\mathrm{td}, J=1.3,8.2 \mathrm{~Hz}$,
$1 \mathrm{H}, \mathrm{Ar}), 7.64(\mathrm{dd}, J=7.9,13.2 \mathrm{~Hz}, 2 \mathrm{H}, \mathrm{Ar}), 8.25(\mathrm{~s}, 1 \mathrm{H}$, Imidazole) ${ }^{13} \mathrm{C}$ NMR (DMSO- $\left.d_{6}\right) \delta: 26.5\left(\mathrm{NCH}_{2} \mathrm{CH}_{2}\right)$, $40.7\left(\mathrm{NCH}_{2}\right), 110.8(\mathrm{CH}), 119.9(\mathrm{CH}), 122.2(\mathrm{CH}), 123.0$ $(\mathrm{CH}), 133.9(\mathrm{C}), 143.5(\mathrm{C}), 144.5$ (CH-imidazole), 161.9 (C), 178.2 (C). HRMS (ESI) $\mathrm{m} / z$ Calculated: 246.0575 $[\mathrm{M}+\mathrm{H}]^{+}$, Found: $246.0583[\mathrm{M}+\mathrm{H}]^{+}$. 


\section{Protein-Ligand Contacts}

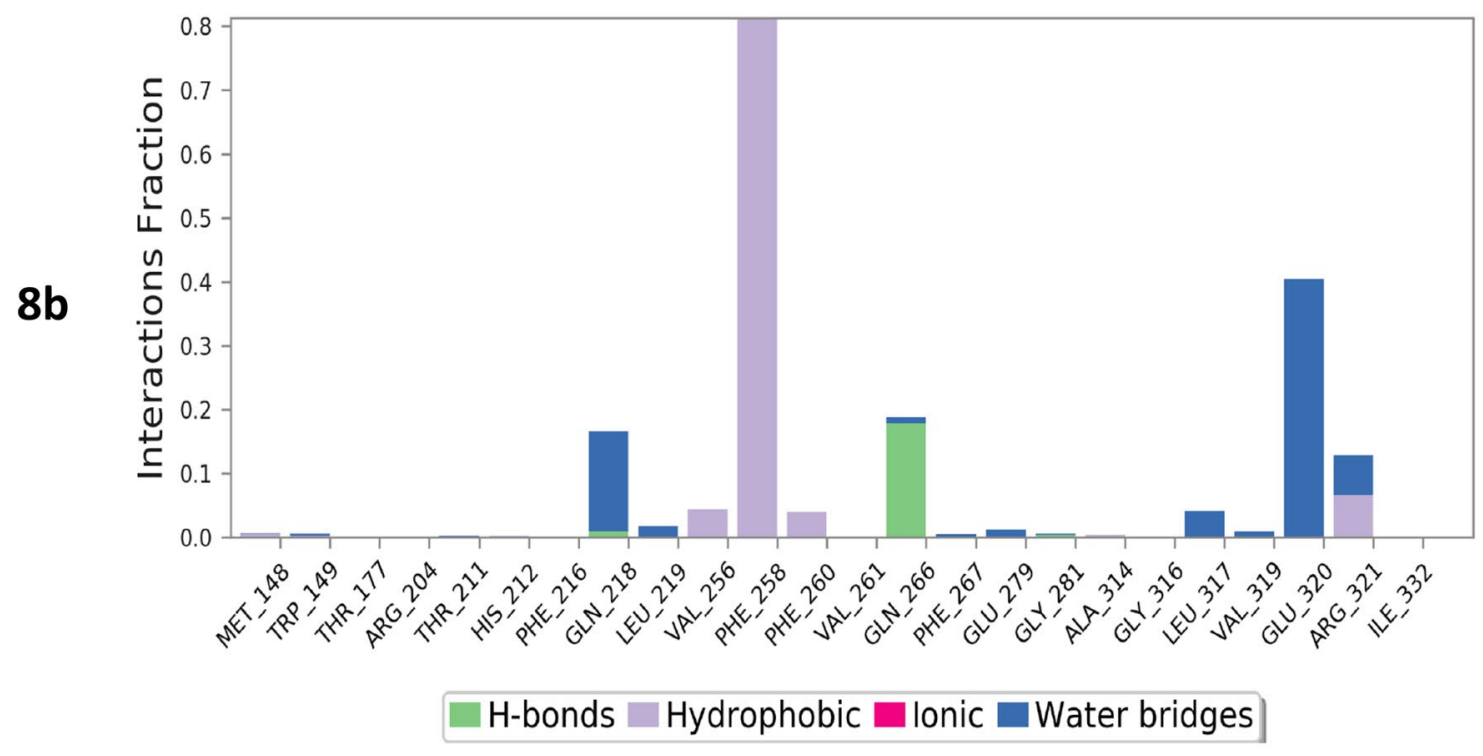

Protein-Ligand Contacts

$9 b$

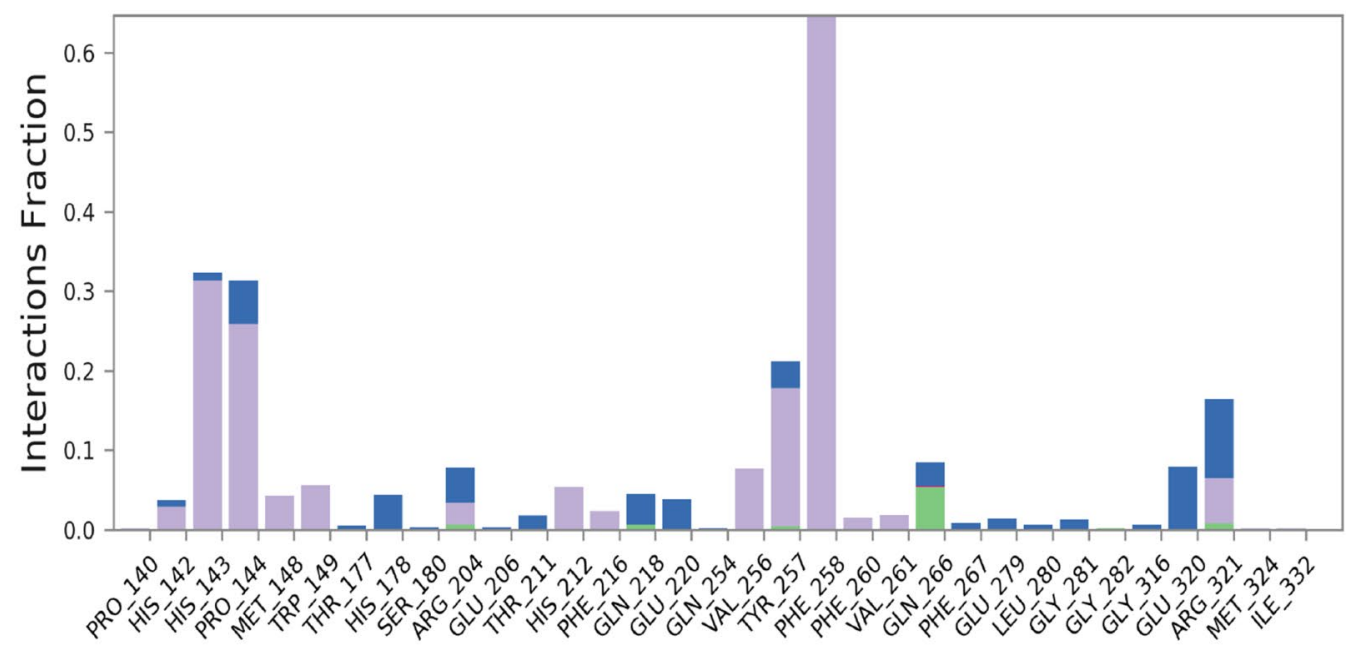

H-bonds Hydrophobic $\square$ lonic $\square$ Water bridges

Fig. 6 continued

\section{5- [2-(1H-benzo[d]imidazol-1-yl)}

\section{ethyl]-1,3,4-thiadiazole-2-thiol (7)}

A mixture of 3-(1H-benzo[d]imidazol-1-yl)propane hydrazide (3) $(1 \mathrm{~g}, 4.9 \mathrm{mmol})$ and $\mathrm{KOH}(0.27 \mathrm{~g}$, $4.9 \mathrm{mmol})$ in absolute $\mathrm{EtOH}(12 \mathrm{~mL})$ was allowed to stir at room temperature for $30 \mathrm{~min}$, then $\mathrm{CS}_{2}(0.56 \mathrm{~g}$, $7.35 \mathrm{mmol}$ ) was added. The reaction mixture was heated under reflux overnight. The solvent was removed under vacuum and concentrated $\mathrm{H}_{2} \mathrm{SO}_{4}(25 \mathrm{~mL})$ was added and the mixture was allowed to stir for $4 \mathrm{~h}$. The reaction mixture was poured into crushed ice and stirred for $1 \mathrm{~h}$, then the resulting solid was collected by filtration, washed several times with $\mathrm{H}_{2} \mathrm{O}$ and dried under vacuum at $40{ }^{\circ} \mathrm{C}$ to obtain the product as a beige solid: Yield: $0.78 \mathrm{~g}(60 \%)$; mp $232-234{ }^{\circ} \mathrm{C}$; HPLC: $100 \%$ at R.T. 3.28 min. ${ }^{1} \mathrm{H}$ NMR (DMSO- $d_{6}$ ) $\delta: \mathrm{CH}_{2}$ peak is obscured by $\mathrm{H}_{2} \mathrm{O}$ of DMSO- $d_{6}$ 


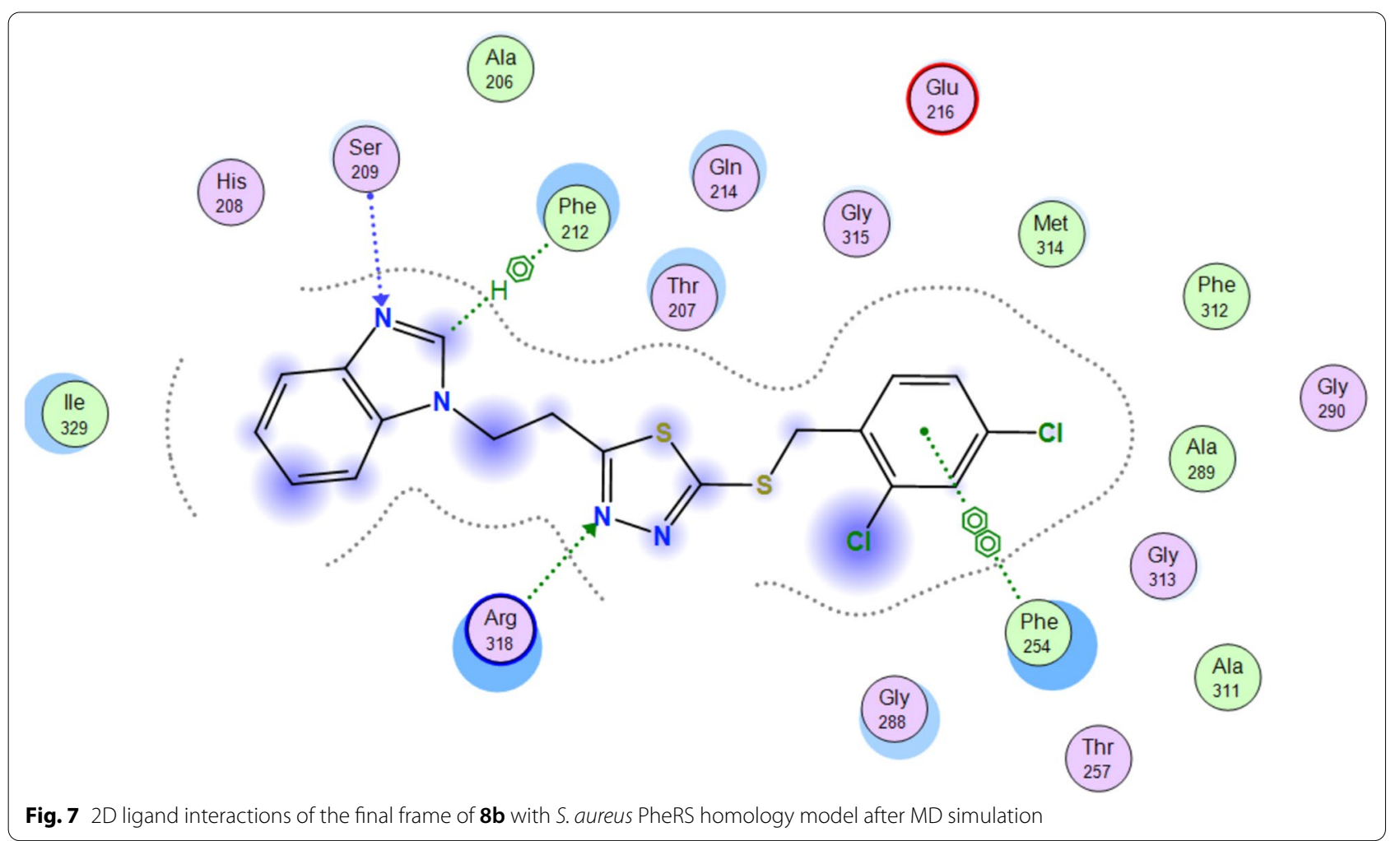

Fig. 7 2D ligand interactions of the final frame of $\mathbf{8 b}$ with S. aureus PheRS homology model after MD simulation

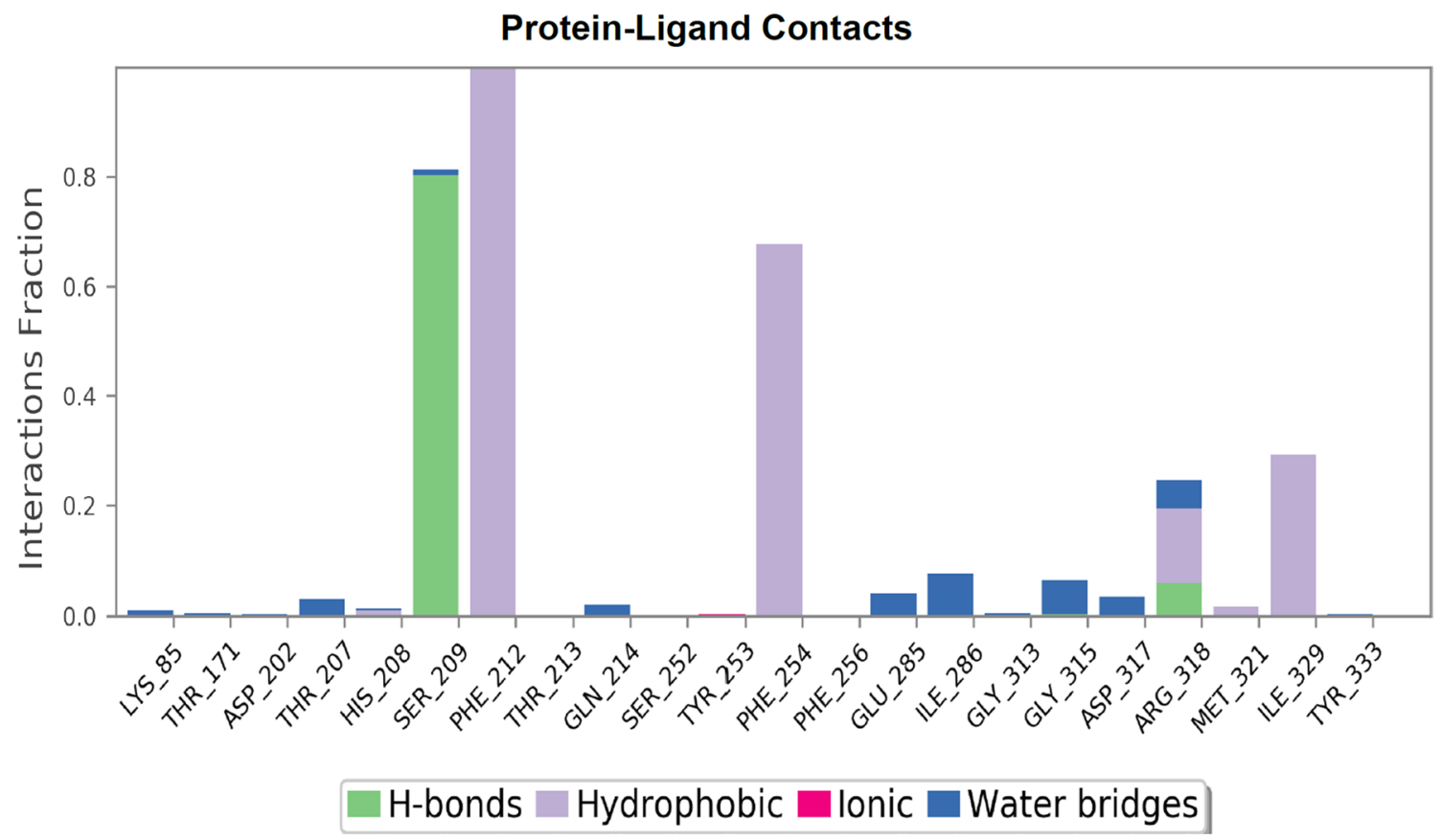

Fig. 8 Diagram showing the S. aureus PheRS homology model_compound $\mathbf{8 b}$ interactions throughout the $200 \mathrm{~ns}$ simulation. The stacked bar charts are normalised over the course of the trajectory, for example; a value of 0.4 indicates the specific reaction is maintained $40 \%$ of the simulation time 


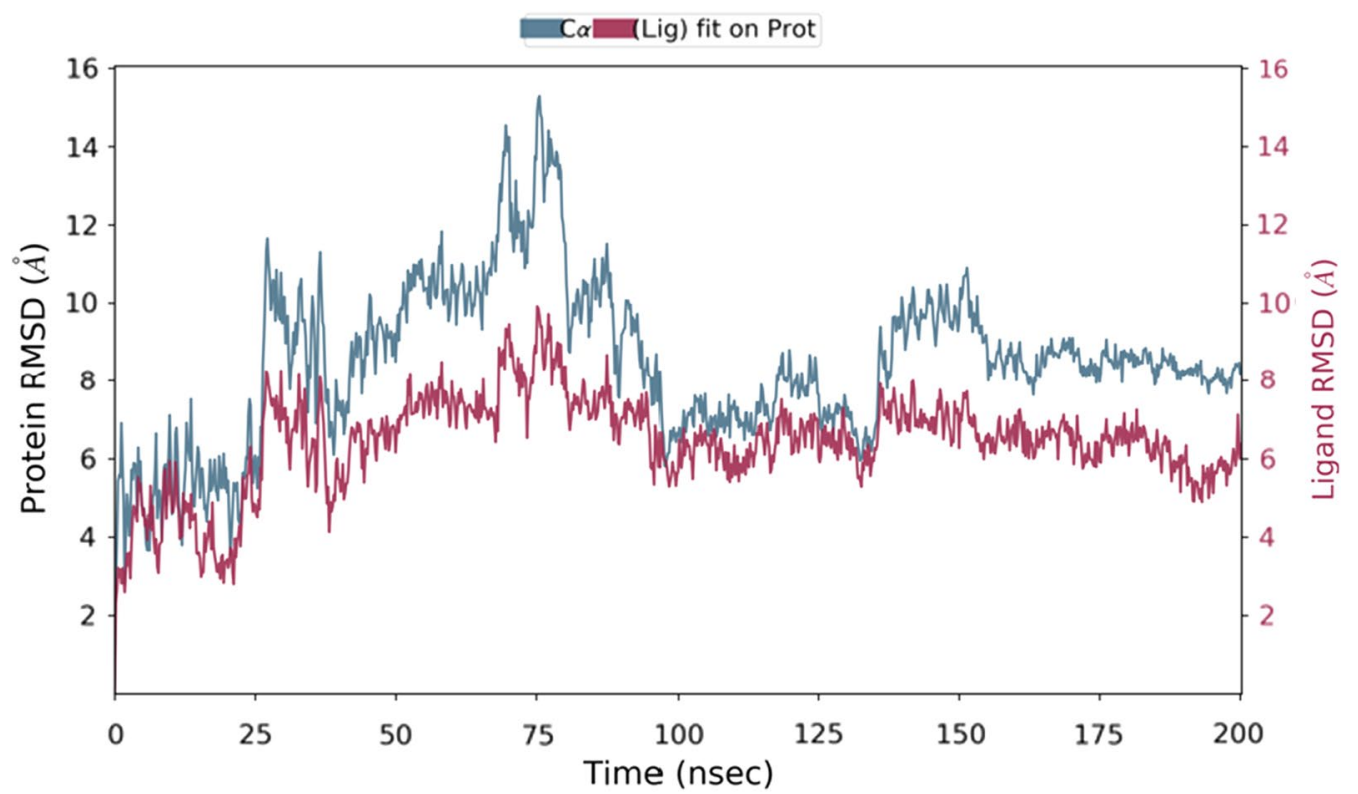

Fig. 9 RMSD $[\AA]$ plot with respect to time in nanoseconds during 200 ns MD simulation of S. aureus homology model and compound 8 b complex

peak, $4.80\left(\mathrm{t}, J=6.7 \mathrm{~Hz}, 2 \mathrm{H}, \mathrm{NCH}_{2}\right), 7.46(\mathrm{~m}, 2 \mathrm{H}, \mathrm{Ar})$, $7.78(\mathrm{~d}, J=7.3 \mathrm{~Hz}, 1 \mathrm{H}, \mathrm{Ar}), 7.88(\mathrm{~d}, J=7.5 \mathrm{~Hz}, 1 \mathrm{H}, \mathrm{Ar})$, 9.01 (s, 1H, Imidazole). ${ }^{13} \mathrm{C}$ NMR (DMSO- $\left.d_{6}\right) \delta: 25.9$ $\left(\mathrm{NCH}_{2} \underline{\mathrm{CH}}_{2}\right), 41.9\left(\mathrm{NCH}_{2}\right), 112.4(\mathrm{CH}), 117.4(\mathrm{CH}), 124.9$ $(\mathrm{CH}), 125.1(\mathrm{CH}), 132.4(\mathrm{C}), 136.6(\mathrm{C}), 143.5$ (CH-imidazole), 155.1 (C). HRMS (ESI) $\mathrm{m} / z$ Calculated: 262.0347 $[\mathrm{M}+\mathrm{H}]^{+}$, Found: $262.0387[\mathrm{M}+\mathrm{H}]^{+}$

\section{General method for the preparation of 2-(2- $(1 \mathrm{H}-$ benzo[d] imidazol-1-yl)ethyl)-5-(arylthio)-1,3,4-oxadiazole (5a-d, 6a-c) and 2-(2-(1H-benzo[d]imidazol-1-yl) ethyl)-5-(arylthio)-1,3,4-thiadiazole (8a-d, 9a-c)}

A mixture of aromatic thiol ( 4 or 7 ) (1 equivalent) and $\mathrm{K}_{2} \mathrm{CO}_{3}$ (1.5 equivalent) in anhydrous DMF (8 $\mathrm{mL} / 0.89 \mathrm{mmol})$ was allowed to stir at room temperature for $30 \mathrm{~min}$. Then the appropriate alkyl halide (1.1 equivalent) in anhydrous DMF $(4 \mathrm{~mL} / 0.89 \mathrm{mmol})$ was added and the reaction mixture allowed to stir at room temperature overnight. The solvent was removed under vacuum and then $\mathrm{H}_{2} \mathrm{O}(50 \mathrm{~mL} / 0.89 \mathrm{mmol})$ was added, and the mixture extracted with chloroform $(3 \times 50 \mathrm{~mL} / 0.89 \mathrm{mmol})$. The combined organic layers were dried $\left(\mathrm{MgSO}_{4}\right)$ and evaporated, then the product was purified using gradient column chromatography.

2 - \{[2 - (1H-benzo[d]imidazol-1-yl)ethyl]5-(benzylthio)\}-1,3,4-oxadiazole (5a).

Prepared from 5-(2-(1H-benzo[d]imidazol-1-yl) ethyl)-1,3,4-oxadiazole-2-thiol (4) (0.2 g, $0.813 \mathrm{mmol}$ ) and benzyl chloride $(0.11 \mathrm{~g}, 0.89 \mathrm{mmol})$. Purified using gradient chromatography eluting with $100 \%$ EtOAc to afford the product as a white solid: Yield: $0.16 \mathrm{~g}(59 \%)$; mp 80-82 ${ }^{\circ} \mathrm{C}$; TLC: $100 \%$ EtOAc, $\mathrm{R}_{\mathrm{f}}$ 0.22; HPLC: $100 \%$ at R.T. $4.25 \mathrm{~min} .{ }^{1} \mathrm{H}$ NMR (DMSO- $\left.d_{6}\right) \delta: 3.44(\mathrm{t}, J=6.8 \mathrm{~Hz}$, $\left.2 \mathrm{H}, \mathrm{NCH}_{2} \mathrm{CH}_{2}\right), 4.40\left(\mathrm{~s}, 2 \mathrm{H}, \mathrm{SCH}_{2}\right), 4.67(\mathrm{t}, J=6.7 \mathrm{~Hz}$, 2H, $\mathrm{NCH}_{2}$ ), 7.19-7.34 (m, 5H, Ar), 7.37 (m, 2H, Ar), 7.57 (dd, $J=1.2,7.3 \mathrm{~Hz}, 1 \mathrm{H}, \mathrm{Ar}$ ), 7.64 (dd, $J=0.9,7 \mathrm{~Hz}, 1 \mathrm{H}$, Ar), 8.19 (s, $1 \mathrm{H}, \mathrm{CH}$-imidazole). ${ }^{13} \mathrm{C}$ NMR (DMSO- $\left.d_{6}\right) \delta$ : $26.2\left(\mathrm{NCH}_{2} \mathrm{CH}_{2}\right), 36.1\left(\mathrm{SCH}_{2}\right), 41.2\left(\mathrm{NCH}_{2}\right), 110.7(\mathrm{CH})$, $120.0(\mathrm{CH}), 122.1(\mathrm{CH}), 122.9(\mathrm{CH}), 128.2(\mathrm{CH}), 129.0$ (2xCH), $129.4(2 \times C H), 133.9(\mathrm{C}), 136.9(\mathrm{C}), 143.8(\mathrm{C})$, 144.5 (CH-imidazole), 163.6 (C), 165.9 (C). HRMS (ESI) $\mathrm{m} / z$ Calculated: $336.1045[\mathrm{M}+\mathrm{H}]^{+}$, Found: 336.1056 $[\mathrm{M}+\mathrm{H}]^{+}$.

2-\{[2-(1H-benzo[d]imidazol-1-yl)ethyl]-5- [(2,4-dichlorobenzyl)thio]\}-1,3,4-oxadiazole (5b).

Prepared from 5-(2-(1H-benzo[d]imidazol-1-yl) ethyl)-1,3,4-oxadiazole-2-thiol (4) (0.2 g, $0.813 \mathrm{mmol})$ and 2,4-dichlorobenzyl chloride (0.17 g, $0.89 \mathrm{mmol})$. Purified using gradient chromatography eluting with $100 \%$ EtOAc to afford the product as a white solid: Yield: 0.26 g (79\%); mp 86-88 ${ }^{\circ} \mathrm{C}$; TLC: $100 \%$ EtOAc, $\mathrm{R}_{\mathrm{f}} 0.36$; HPLC: $100 \%$ at R.T. 4.62 min. ${ }^{1} \mathrm{H}$ NMR (DMSO- $d_{6}$ ) $\delta$ : $3.44\left(\mathrm{t}, J=6.8 \mathrm{~Hz}, 2 \mathrm{H}, \mathrm{NCH}_{2} \mathrm{CH}_{2}\right), 4.47$ (s, $2 \mathrm{H}, \mathrm{SCH}_{2}$ ), $4.67\left(\mathrm{t}, J=6.8 \mathrm{~Hz}, 2 \mathrm{H}, \mathrm{NCH}_{2}\right), 7.21$ (m, 2H, Ar), 7.38 (dd, $J=2.2,8.3 \mathrm{~Hz}, 1 \mathrm{H}, \mathrm{Ar}), 7.49$ (d, $J=8.4 \mathrm{~Hz}, 1 \mathrm{H}, \mathrm{Ar}), 7.56$ (dd, $J=1.7,7.2 \mathrm{~Hz}, 1 \mathrm{H}, \mathrm{Ar}), 7.64(\mathrm{~m}, 2 \mathrm{H}, \mathrm{Ar}), 8.21(\mathrm{~s}, 1 \mathrm{H}$, imidazole). ${ }^{13} \mathrm{C}$ NMR (DMSO- $\left.d_{6}\right) \delta: 26.3\left(\mathrm{NCH}_{2} \mathrm{CH}_{2}\right)$, $33.9\left(\mathrm{SCH}_{2}\right), 41.2\left(\mathrm{NCH}_{2}\right), 110.6(\mathrm{CH}), 120.0(\mathrm{CH}), 122.1$ $(\mathrm{CH}), 122.9(\mathrm{CH}), 128.0(\mathrm{CH}), 129.6(\mathrm{CH}), 133.2(\mathrm{CH})$, 133.5 (C), 133.9 (C), 134.0 (C), 134.7 (C), 143.8 (C), 


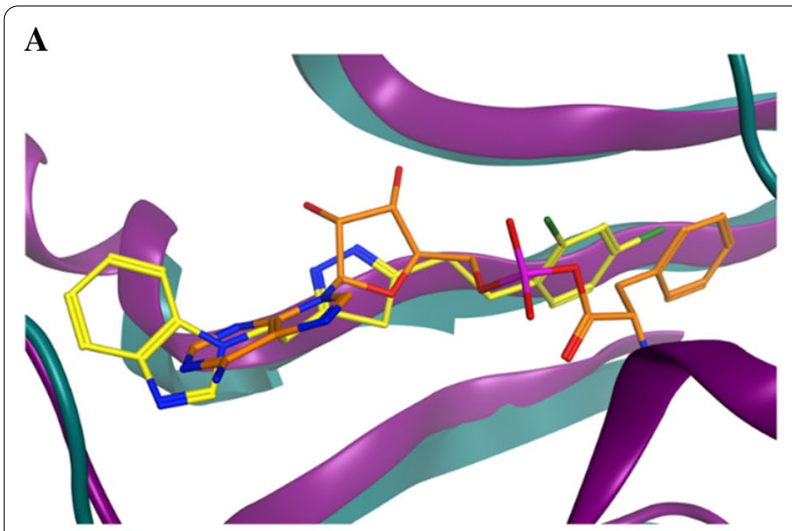

B

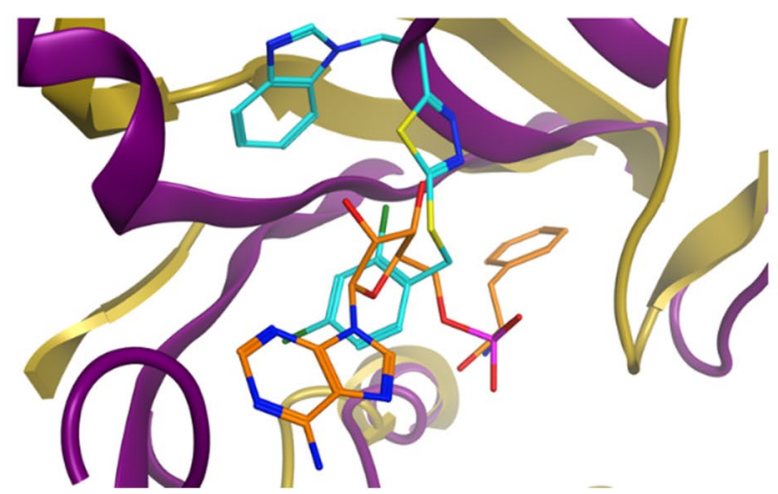

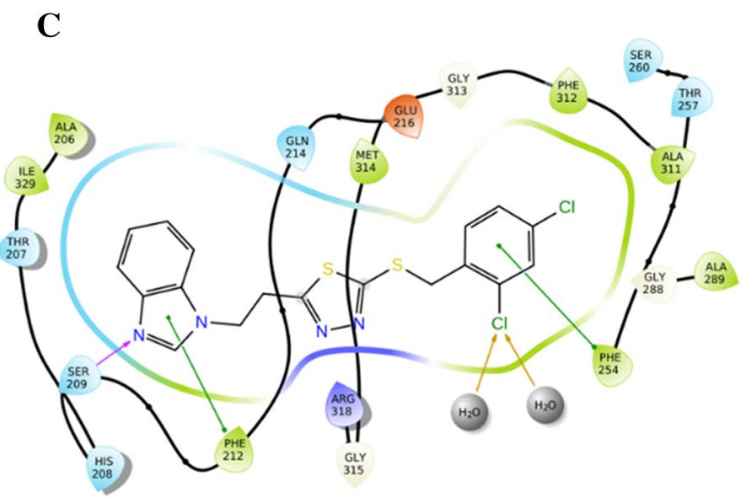

D

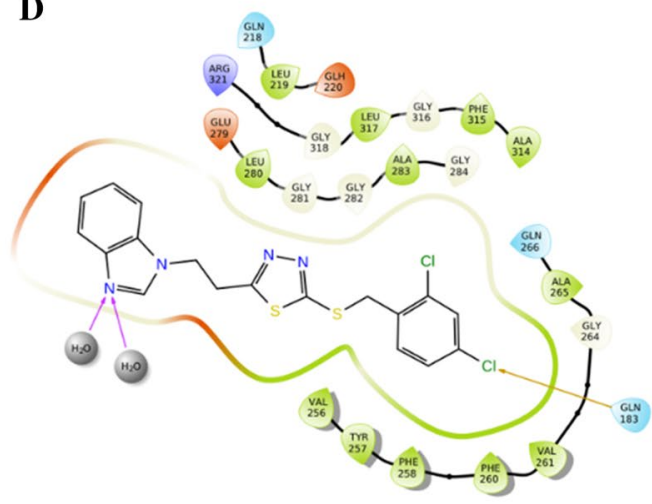

Fig. 10 Illustrating the difference between $\mathbf{8 b}$ interactions with Th. thermophilus PheRS and S. aureus PheRS. A) The purple ribbon is Th. thermophilus PheRS co-crystallised with Phe-AMP (orange colour) superimposed with the S. aureus PheRS homology model (green ribbon) and the MD simulation final frame of $\mathbf{8 b}$ (yellow colour), B) The purple ribbon is Th. thermophilus PheRS co-crystallised with Phe-AMP (orange colour) superimposed with the Th. thermophilus PheRS (golden ribbon) and the MD simulation final frame of $\mathbf{8 b}$ (light blue colour), C) the 2D ligand interactions of the MD simulation final frame of $\mathbf{8} \mathbf{b}$ with S. aureus PheRS homology model, D) the 2D ligand interactions of the MD simulation final frame of $\mathbf{8} \mathbf{b}$ with Th. thermophilus PheRS

144.6 (CH-imidazole), 162.9 (C), 166.2 (C). HRMS (ESI) $\mathrm{m} / \mathrm{z}$ Calculated: $404.0265[\mathrm{M}+\mathrm{H}]^{+}$, Found: 404.0276 $[\mathrm{M}+\mathrm{H}]^{+}$.

2-\{[2-(1H-benzo[d]imidazol-1-yl)ethyl]-5[(4-fluorobenzyl)thio]\}-1,3,4-oxadiazole (5c).

Prepared from 5-(2-(1H-benzo[d]imidazol-1-yl) ethyl)-1,3,4-oxadiazole-2-thiol (4) (0.2 g, $0.813 \mathrm{mmol})$ and 4-flourobenzyl chloride $(0.13 \mathrm{~g}, 0.89 \mathrm{mmol})$. Purified using gradient chromatography eluting with $100 \%$ EtOAc to afford the product as a white semisolid: Yield: $0.205 \mathrm{~g}$ (71\%); TLC: $100 \%$ EtOAc, $\mathrm{R}_{\mathrm{f}} 0.20$; HPLC: $100 \%$ at R.T. 4.28 min. ${ }^{1} \mathrm{H}$ NMR (DMSO- $\left.d_{6}\right) \delta: 3.44(\mathrm{t}, J=6.7 \mathrm{~Hz}$, $\left.2 \mathrm{H}, \mathrm{NCH}_{2} \mathrm{CH}_{2}\right), 4.40\left(\mathrm{~s}, 2 \mathrm{H}, \mathrm{SCH}_{2}\right), 4.67(\mathrm{t}, J=6.7 \mathrm{~Hz}$, $\left.2 \mathrm{H}, \mathrm{NCH}_{2}\right), 7.14(\mathrm{t}, J=8.9 \mathrm{~Hz}, 2 \mathrm{H}, \mathrm{Ar}), 7.22(\mathrm{~m}, 2 \mathrm{H}, \mathrm{Ar})$, $7.42(\mathrm{~m}, 2 \mathrm{H}, \mathrm{Ar}), 7.57$ (dd, $J=1.3,7.3 \mathrm{~Hz}, 1 \mathrm{H}, \mathrm{Ar}), 7.64$ (dd, $J=1,6.9 \mathrm{~Hz}, 1 \mathrm{H}, \mathrm{Ar}), 8.19$ (s, $1 \mathrm{H}$, imidazole). ${ }^{13} \mathrm{C}$ NMR (DMSO- $\left.d_{6}\right) \delta$ : $26.2\left(\mathrm{NCH}_{2} \mathrm{CH}_{2}\right), 35.3\left(\mathrm{SCH}_{2}\right), 41$. $\left(\mathrm{NCH}_{2}\right), 110.7(\mathrm{CH}), 115.7(\mathrm{CH}), 115.9(\mathrm{CH}), 120.0(\mathrm{CH})$, $122.1(\mathrm{CH}), 122.9(\mathrm{CH}), 131.5(\mathrm{CH}), 131.6(\mathrm{CH}), 131.3$ (C), 133.9 (C), 143.8, (C), 144.6 (CH-imidazole), 161.1
(C), 163.0 (C), 163.5 (C), 165.9 (C). HRMS (ESI): Calculated: $354.0951[\mathrm{M}+\mathrm{H}]^{+}$, Found: $354.0961[\mathrm{M}+\mathrm{H}]^{+}$.

4-\{[( 5- [2-(1H-benzo[d]imidazol-1-yl)ethyl]-1,3,4-oxadiazol-2-yl)\}thio)methyl] benzonitrile (5d).

Prepared from 5-(2-(1H-benzo[d]imidazol-1-yl) ethyl)-1,3,4-oxadiazole-2-thiol (4) (0.2 g, $0.813 \mathrm{mmol})$ and 4-(chloromethyl)benzonitrile $(0.14 \mathrm{~g}, 0.89 \mathrm{mmol})$. Purified using gradient chromatography eluting with $100 \%$ EtOAc to afford the product as a white semisolid: Yield: $0.195 \mathrm{~g}$ (67\%); TLC: $100 \%$ EtOAc, $\mathrm{R}_{\mathrm{f}}$ 0.36; HPLC: $100 \%$ at R.T. 4.02 min. ${ }^{1} \mathrm{H}$ NMR (DMSO- $\left.d_{6}\right) \delta: 3.43(\mathrm{t}$, $\left.J=6.8 \mathrm{~Hz}, 2 \mathrm{H}, \mathrm{NCH}_{2} \mathrm{CH}_{2}\right), 4.48\left(\mathrm{~s}, 2 \mathrm{H}, \mathrm{SCH}_{2}\right), 4.66(\mathrm{t}$, $\left.J=6.7 \mathrm{~Hz}, 2 \mathrm{H}, \mathrm{NCH}_{2}\right), 7.21(\mathrm{~m}, 2 \mathrm{H}, \mathrm{Ar}), 7.56(\mathrm{~m}, 3 \mathrm{H}, \mathrm{Ar})$, 7.64 (m, 1H, Ar), 7.77 (d, J=8.4 Hz, 2H, Ar), $8.19(\mathrm{~s}, 1 \mathrm{H}$, imidazole). ${ }^{13} \mathrm{C}$ NMR (DMSO- $\left.d_{6}\right) \delta: 26.2\left(\mathrm{NCH}_{2} \mathrm{CH}_{2}\right)$, $35.5\left(\mathrm{SCH}_{2}\right), 41.2\left(\mathrm{NCH}_{2}\right), 110.6(\mathrm{CH}), 110.9(\mathrm{CN}), 119.1$ (C), $119.9(\mathrm{CH}), 122.1(\mathrm{CH}), 122.9(\mathrm{CH}), 130.4(2 \times C H)$, $132.9(2 \times C H), 133.9(\mathrm{C}), 143.2,(\mathrm{C}), 143.8(\mathrm{C}), 144.6(\mathrm{CH}-$ imidazole), 163.2 (C), 166.1 (C). HRMS (ESI): Calculated: $361.0997[\mathrm{M}+\mathrm{H}]^{+}$, Found: $361.1004[\mathrm{M}+\mathrm{H}]^{+}$. 
<smiles>[R]c1ccc(CSc2nnc(CCn3cnc4ccccc43)s2)cc1</smiles>

25-(phenethylthio)\}-1,3,4-oxadiazole (6a).

Prepared from 5-(2-(1H-benzo[d]imidazol-1-yl) ethyl)-1,3,4-oxadiazole-2-thiol (4) $(0.2 \mathrm{~g}, 0.813 \mathrm{mmol})$

Table 1 Yields and melting points (m.p.) of compounds $\mathbf{5 a - d}$ 6a-c, 8a-d and $9 a-c$

\begin{tabular}{|c|c|c|c|c|c|}
\hline \multicolumn{3}{|l|}{ Series 1} & \multicolumn{3}{|l|}{ Series 2} \\
\hline Compounds & $\%$ yield & m.p. $\left({ }^{\circ} \mathrm{C}\right)$ & Compounds & $\%$ yield & m.p. $\left({ }^{\circ} \mathrm{C}\right)$ \\
\hline $5 a$ & 59 & $80-82$ & $8 a$ & 62 & $88-90$ \\
\hline $5 b$ & 79 & $86-88$ & $8 b$ & 69 & $84-86$ \\
\hline $5 c$ & 71 & semisolid & $8 c$ & 71 & $76-78$ \\
\hline $5 d$ & 67 & semisolid & $8 d$ & 61 & $112-114$ \\
\hline $6 a$ & 60 & $78-80$ & $9 a$ & 51 & $76-78$ \\
\hline $6 b$ & 65 & $90-92$ & $9 b$ & 40 & $96-98$ \\
\hline $6 c$ & 75 & $65-67$ & $9 c$ & 55 & $68-70$ \\
\hline
\end{tabular}

and 2-bromomethyl benzene (0.166 g, $0.89 \mathrm{mmol})$. Purified using gradient chromatography eluting with $\mathrm{CH}_{2} \mathrm{Cl}_{2}-\mathrm{MeOH} 97.5: 2.5 \mathrm{v} / \mathrm{v}$ to afford the product as a white semisolid: Yield: $0.166 \mathrm{~g}(62 \%)$; mp $78-80{ }^{\circ} \mathrm{C}$; TLC: $\mathrm{CH}_{2} \mathrm{Cl}_{2}-\mathrm{MeOH}$ 9:1 v/v, $\mathrm{R}_{\mathrm{f}}$ 0.55; HPLC: $100 \%$ at $\mathrm{RT}=4.35 \mathrm{~min} .{ }^{1} \mathrm{H}$ NMR (DMSO- $\left.d_{6}\right) \delta: 2.96(\mathrm{t}, J=7.2 \mathrm{~Hz}$ $\left.2 \mathrm{H}, \mathrm{SCH}_{2} \mathrm{CH}_{2}\right), \mathrm{CH}_{2}$ signal is obscured by $\mathrm{H}_{2} \mathrm{O}$ in DMSO- $d_{6}$ signal, $3.44\left(\mathrm{t}, J=6.7 \mathrm{~Hz}, 2 \mathrm{H}, \mathrm{SCH}_{2}\right), 4.69$ $\left(\mathrm{t}, J=6.7 \mathrm{HzH}, \mathrm{NCH}_{2}\right), 7.19-7.31(\mathrm{~m}, 7 \mathrm{H}, \mathrm{Ar}), 7.59(\mathrm{~d}$, $J=7.9 \mathrm{~Hz}, 1 \mathrm{H}, \mathrm{Ar}), 7.63(\mathrm{~d}, J=7.8 \mathrm{~Hz}, 1.0 \mathrm{~Hz}, 1 \mathrm{H}, \mathrm{Ar})$, 8.22 (s, $1 \mathrm{H}$, imidazole). ${ }^{13} \mathrm{C}$ NMR (DMSO- $\left.d_{6}\right) \delta: 26.2$ $\left(\mathrm{NCH}_{2} \underline{\mathrm{CH}}_{2}\right), 33.6\left(\mathrm{SCH}_{2}\right), 35.2\left(\mathrm{SCH}_{2} \underline{\mathrm{C}} \mathrm{H}_{2}\right), 41.3\left(\mathrm{NCH}_{2}\right)$, $110.7(\mathrm{CH}), 120.0(\mathrm{CH}), 122.2(\mathrm{CH}), 122.8(\mathrm{CH}), 127.0$ $(\mathrm{CH}), 128.9(2 \times \mathrm{CH}), 129.1(2 \times \mathrm{CH}), 133.9(\mathrm{C}), 139.6(\mathrm{C})$, 143.8 (C), 144.6 (CH-imidazole), 163.9 (C), 165.7 (C). HRMS (ESI) $m / z$ Calculated: $350.1201[\mathrm{M}+\mathrm{H}]^{+}$, Found: $350.1211[\mathrm{M}+\mathrm{H}]^{+}$. 
2- $\{[2-(1 H-b e n z o[d]$ imidazol-1-yl)ethyl]-5- [(4-chlorophenethyl)thio]\}-1,3,4-oxadiazole (6b).

Prepared from 5-(2-(1H-benzo[d]imidazol-1-yl) ethyl)-1,3,4-oxadiazole-2-thiol (4) (0.2 g, $0.813 \mathrm{mmol})$ and 4-chlorophenethyl bromide $(0.196 \mathrm{~g}, 0.89 \mathrm{mmol})$. Purified using gradient chromatography eluting with $100 \%$ EtOAc to afford the product as a white solid: Yield: $0.203 \mathrm{~g}$ (65\%); mp 90-92 ${ }^{\circ} \mathrm{C}$; TLC: $\mathrm{CH}_{2} \mathrm{Cl}_{2}-\mathrm{MeOH}$ 9:1 v/v, $\mathrm{R}_{\mathrm{f}}$ 0.57; HPLC: $100 \%$ at R.T. $4.50 \mathrm{~min} .{ }^{1} \mathrm{H}$ NMR (DMSO$\left.d_{6}\right) \delta: 2.96\left(\mathrm{t}, J=7.4 \mathrm{~Hz}, 2 \mathrm{H}, \mathrm{SCH}_{2} \mathrm{CH}_{2}\right), \mathrm{CH}_{2}$ signal is obscured by $\mathrm{H}_{2} \mathrm{O}$ in DMSO- $d_{6}$ signal, $3.44(\mathrm{t}, J=6.7 \mathrm{~Hz}$, $\left.2 \mathrm{H}, \mathrm{SCH}_{2}\right), 4.68\left(\mathrm{t}, J=6.7 \mathrm{~Hz}, 2 \mathrm{H}, \mathrm{NCH}_{2}\right), 7.22(\mathrm{~m}, 4 \mathrm{H}$, Ar), $7.35(\mathrm{~d}, J=8.4 \mathrm{~Hz}, 2 \mathrm{H}, \mathrm{Ar}), 7.58(\mathrm{~d}, J=8.1 \mathrm{~Hz}, 1 \mathrm{H}$, Ar), 7.63 (d, $J=7.8 \mathrm{~Hz}, 1 \mathrm{H}, \mathrm{Ar}), 8.22(\mathrm{~s}, 1 \mathrm{H}, \mathrm{CH}$-imidazole). ${ }^{13} \mathrm{C}$ NMR (DMSO- $\left.d_{6}\right) \delta: 26.2\left(\mathrm{NCH}_{2} \mathrm{CH}_{2}\right), 33.3$ $\left(\mathrm{SCH}_{2}\right), 34.5\left(\mathrm{SCH}_{2} \mathrm{CH}_{2}\right), 41.3\left(\mathrm{NCH}_{2}\right), 110.7(\mathrm{CH})$, $120.0(\mathrm{CH}), 122.1(\mathrm{CH}), 122.9(\mathrm{CH}), 128.8(2 \times \mathrm{CH})$, 131.0 (2xCH), 131.7 (C), 133.9 (C), $138.6(\mathrm{C}), 143.8(\mathrm{C})$, 144.6 (CH-imidazole), 163.8 (C), 165.8 (C). HRMS (ESI) $\mathrm{m} / z$ Calculated: $384.0812[\mathrm{M}+\mathrm{H}]^{+}$, Found: 384.0820 $[\mathrm{M}+\mathrm{H}]^{+}$.

2-\{[2-(1H-benzo[d]imidazol-1-yl)ethyl]-5- [(4-methoxyphenethyl)thio]\}-1,3,4-oxadiazole (6c).

Prepared from 5-(2-(1H-benzo[d]imidazol-1-yl) ethyl)-1,3,4-oxadiazole-2-thiol (4) $(0.2 \mathrm{~g}, 0.813 \mathrm{mmol})$ and 4-methoxyphenethyl bromide $(0.19 \mathrm{~g}, 0.89 \mathrm{mmol})$. Purified using gradient chromatography eluting with $100 \%$ EtOAc to afford the product as a white solid: Yield: $0.2 \mathrm{~g}(75 \%)$; mp $65-67{ }^{\circ} \mathrm{C}$; TLC: $\mathrm{CH}_{2} \mathrm{Cl}_{2}-\mathrm{MeOH}$ 9:1 v/v, $\mathrm{R}_{\mathrm{f}}$ 0.57; HPLC: $100 \%$ at R.T. $4.34 \mathrm{~min} .{ }^{1} \mathrm{H}$ NMR (DMSO- $d_{6}$ ) $\delta: 2.89\left(\mathrm{t}, J=6.8 \mathrm{~Hz}, 2 \mathrm{H}, \mathrm{SCH}_{2} \mathrm{CH}_{2}\right), \mathrm{CH}_{2}$ signal is obscured by $\mathrm{H}_{2} \mathrm{O}$ in DMSO- $d_{6}$ signal, 3.44 $\left(\mathrm{t}, J=6.8 \mathrm{~Hz}, 2 \mathrm{H}, \mathrm{SCH}_{2}\right), 3.73\left(\mathrm{~s}, 3 \mathrm{H}, \mathrm{OCH}_{3}\right), 4.69(\mathrm{t}$, $\left.J=6.7 \mathrm{~Hz}, 2 \mathrm{H}, \mathrm{NCH}_{2}\right), 6.85(\mathrm{~d}, J=8.7 \mathrm{~Hz}, 2 \mathrm{H}, \mathrm{Ar}), 7.13$ (d, $J=8.7 \mathrm{~Hz}, 2 \mathrm{H}, \mathrm{Ar}), 7.20$ (td, $J=1.2,7.3 \mathrm{~Hz}, 1 \mathrm{H}, \mathrm{Ar}$ ), $7.25(\mathrm{td}, J=1.2,8.1 \mathrm{~Hz}, 1 \mathrm{H}, \mathrm{Ar}), 7.59(\mathrm{~d}, J=7.9 \mathrm{~Hz}, 1 \mathrm{H}$, Ar), $7.63(\mathrm{~d}, J=7.7 \mathrm{~Hz}, 1,1 \mathrm{H}, \mathrm{Ar}), 8.22(\mathrm{~s}, 1 \mathrm{H}$, imidazole). ${ }^{13} \mathrm{C}$ NMR (DMSO- $d_{6} \delta: 26.2\left(\mathrm{NCH}_{2} \underline{\mathrm{C}} \mathrm{H}_{2}\right), 33.9$ $\left(\mathrm{SCH}_{2}\right), 34.4\left(\mathrm{SCH}_{2} \mathrm{CH}_{2}\right), 41.3\left(\mathrm{NCH}_{2}\right), 55.9\left(\mathrm{OCH}_{3}\right)$, $110.7(\mathrm{CH}), 114.3(2 \times \mathrm{CH}), 120.0(\mathrm{CH}), 122.1(\mathrm{CH}), 122.9$ (CH), $130.1(2 \times C H), 131.5(\mathrm{C}), 140.0(\mathrm{C}), 143.8(\mathrm{C}), 144.6$ (CH-imidazole), 158.4 (C), 164.0 (C), 165.7 (C). HRMS (ESI) $m / z$ Calculated: $380.130[\mathrm{M}+\mathrm{H}]^{+}$, Found: 380.1320 $[\mathrm{M}+\mathrm{H}]^{+}$.

2 5-(benzylthio)\}-1,3,4-thiadiazole (8a).

Prepared from 5-(2-(1H-benzo[ $[d]$ imidazol-1-yl) ethyl)-1,3,4-thiadiazole-2-thiol (7) $(0.2 \mathrm{~g}, 0.76 \mathrm{mmol})$ and benzyl chloride $(0.11 \mathrm{~g}, 0.84 \mathrm{mmol})$, and purified using gradient chromatography eluting with $\mathrm{CH}_{2} \mathrm{Cl}_{2}-\mathrm{MeOH}$ 97.5:2.5 v/v to afford the as a white solid: Yield: $0.167 \mathrm{~g}$ (62\%); mp 88-90 ${ }^{\circ} \mathrm{C}$; TLC: $\mathrm{CH}_{2} \mathrm{Cl}_{2}-\mathrm{MeOH}$ 9:1 v/v, $\mathrm{R}_{\mathrm{f}}$ 0.5; HPLC: $100 \%$ at R.T. 4.24 min. ${ }^{1} \mathrm{H}$ NMR (DMSO- $d_{6}$ ) $\delta: 3.44\left(\mathrm{t}, J=6.8 \mathrm{~Hz}, 2 \mathrm{H}, \mathrm{NCH}_{2} \mathrm{CH}_{2}\right), 4.40\left(\mathrm{~s}, 2 \mathrm{H}, \mathrm{SCH}_{2}\right)$, $4.67\left(\mathrm{t}, J=6.7 \mathrm{~Hz}, 2 \mathrm{H}, \mathrm{NCH}_{2}\right), 7.19-7.34(\mathrm{~m}, 5 \mathrm{H}, \mathrm{Ar})$, $7.37(\mathrm{~m}, 2 \mathrm{H}, \mathrm{Ar}), 7.57(\mathrm{~m}, 1 \mathrm{H}, \mathrm{Ar}), 7.64(\mathrm{~m}, 1 \mathrm{H}, \mathrm{Ar})$, 8.19 (s, $1 \mathrm{H}$, imidazole). ${ }^{13} \mathrm{C}$ NMR (DMSO- $\left.d_{6}\right) \delta: 26.2$ $\left(\mathrm{NCH}_{2} \mathrm{CH}_{2}\right), 36.1\left(\mathrm{SCH}_{2}\right), 41.3\left(\mathrm{NCH}_{2}\right), 110.7(\mathrm{CH}), 120.0$ $(\mathrm{CH}), 122.1(\mathrm{CH}), 122.9(\mathrm{CH}), 128.2(\mathrm{CH}), 129.0(2 \times C H)$, 129.4 (2xCH), 133.9 (C), $136.9(\mathrm{C}), 143.8(\mathrm{C}), 144.5(\mathrm{CH}-$ imidazole), 163.6 (C), 165.9 (C). HRMS (ESI): Calculated: $352.0816[\mathrm{M}+\mathrm{H}]^{+}$, Found: $352.0850[\mathrm{M}+\mathrm{H}]^{+}$.

2- \{[2-(1H-benzo[d]imidazol-1-yl)ethyl]-5- [(2,4-dichlo-

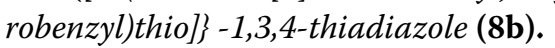

Prepared from 5-(2-(1H-benzo[d]imidazol-1-yl) ethyl)-1,3,4-thiadiazole-2-thiol (7) (0.2 g, $0.76 \mathrm{mmol})$ and 2,4-dichlorobenzyl chloride $(0.16 \mathrm{~g}, 0.84 \mathrm{mmol})$. Purified using gradient chromatography eluting with $\mathrm{CH}_{2} \mathrm{Cl}_{2}-\mathrm{MeOH} 96: 4 \mathrm{v} / \mathrm{v}$ to afford the product as a white solid: Yield: $0.22 \mathrm{~g}$ (69\%); $\mathrm{mp} 84-86{ }^{\circ} \mathrm{C}$; TLC: $\mathrm{CH}_{2} \mathrm{Cl}_{2}-\mathrm{MeOH}$ 9:1 v/v, $\mathrm{R}_{\mathrm{f}}$ 0.55; HPLC: $100 \%$ at R.T. $4.60 \mathrm{~min} ;{ }^{1} \mathrm{H}$ NMR (DMSO- $\left.d_{6}\right) \delta: 3.44(\mathrm{t}, J=6.7 \mathrm{~Hz}, 2 \mathrm{H}$, $\left.\mathrm{NCH}_{2} \mathrm{C}_{2}\right), 4.47\left(\mathrm{~s}, 2 \mathrm{H}, \mathrm{SCH}_{2}\right), 4.67(\mathrm{t}, J=6.7 \mathrm{~Hz}, 2 \mathrm{H}$, $\left.\mathrm{NCH}_{2}\right), 7.21(\mathrm{~m}, 2 \mathrm{H}, \mathrm{Ar}), 7.37(\mathrm{dd}, J=8.3 \mathrm{~Hz}, 2.2 \mathrm{~Hz}$, $1 \mathrm{H}, \mathrm{Ar}), 7.49$ (d, J=8.4 Hz, 1H, Ar), $7.56(\mathrm{~m}, 1 \mathrm{H}, \mathrm{Ar})$, $7.63(\mathrm{~m}, 1 \mathrm{H}, \mathrm{Ar}), 7.66(\mathrm{~d}, J=2.2 \mathrm{~Hz}, 1 \mathrm{H}, \mathrm{Ar}), 8.21(\mathrm{~s}, 1 \mathrm{H}$, imidazole). ${ }^{13} \mathrm{C}$ NMR (DMSO- $\left.d_{6}\right) \delta: 26.3\left(\mathrm{NCH}_{2} \mathrm{CH}_{2}\right)$, $33.9\left(\mathrm{SCH}_{2}\right), 41.2\left(\mathrm{NCH}_{2}\right), 110.6(\mathrm{CH}), 120.0(\mathrm{CH}), 122.1$ $(\mathrm{CH}), 122.9(\mathrm{CH}), 128.0(\mathrm{CH}), 129.6(\mathrm{CH}), 133.2(\mathrm{CH})$, 133.5 (C), 133.9 (C), 134.0 (C), 134.7 (C), 143.8 (C), 144.6 (CH-imidazole), 162.9 (C), 166.2 (C). HRMS (ESI) $\mathrm{m} / \mathrm{z}$ Calculated: $420.0037[\mathrm{M}+\mathrm{H}]^{+}$, Found: 420.0035 $[\mathrm{M}+\mathrm{H}]^{+}$.

2- $\{[2-(1 H-b e n z o[d] i m i d a z o l-1-y l) e t h y l]-5-\quad[(4-f l u o r o b e n-$ zyl)thio]\} -1,3,4-thiadiazole (8c).

Prepared from 5-(2-(1H-benzo[d]imidazol-1-yl) ethyl)-1,3,4-thiadiazole-2-thiol (7) $(0.2 \mathrm{~g}, 0.76 \mathrm{mmol})$ and 4-flourobenzyl chloride $(0.12 \mathrm{~g}, 0.84 \mathrm{mmol})$. Purified using gradient chromatography eluting with $100 \%$ EtOAc to afford the product as a white solid: Yield: $0.16 \mathrm{~g}$ (71\%); mp 76-78 ${ }^{\circ} \mathrm{C}$; TLC: $\mathrm{CH}_{2} \mathrm{Cl}_{2}-\mathrm{MeOH} 9: 1 \mathrm{v} / \mathrm{v}, \mathrm{R}_{\mathrm{f}} 0.62$; HPLC: $100 \%$ at R.T. 4.27 min. ${ }^{1} \mathrm{H}$ NMR (DMSO- $d_{6}$ ) $\delta$ : $3.44\left(\mathrm{t}, J=6.8 \mathrm{~Hz}, 2 \mathrm{H}, \mathrm{NCH}_{2} \mathrm{C}_{2}\right), 4.40\left(\mathrm{~s}, 2 \mathrm{H}, \mathrm{SCH}_{2}\right)$, $4.67\left(\mathrm{t}, J=6.7 \mathrm{~Hz}, 2 \mathrm{H}, \mathrm{NCH}_{2}\right), 7.14(\mathrm{t}, J=8.9 \mathrm{~Hz}, 2 \mathrm{H}, \mathrm{Ar})$, $7.22(\mathrm{~m}, 2 \mathrm{H}, \mathrm{Ar}), 7.41$ (m, 2H, Ar), $7.56(\mathrm{~m}, 1 \mathrm{H}, \mathrm{Ar}), 7.64$ (m, 1H, Ar), 8.19 (s, $1 \mathrm{H}$, Imidazole). ${ }^{13} \mathrm{C}$ NMR (DMSO$\left.d_{6}\right) \delta: 26.2\left(\mathrm{NCH}_{2} \underline{\mathrm{CH}_{2}}\right), 35.3\left(\mathrm{SCH}_{2}\right), 41.2\left(\mathrm{NCH}_{2}\right), 110.7$ $(\mathrm{CH}), 115.7(\mathrm{CH}), 115.9(\mathrm{CH}), 119.9(\mathrm{CH}), 122.1(\mathrm{CH})$, $122.9(\mathrm{CH}), 131.5(\mathrm{CH}), 131.6(\mathrm{CH}), 131.3(\mathrm{C}), 133.9$ (C), $143.8(\mathrm{C}), 144.6$ (CH-imidazole), 161.1 (C), 163.0 (C), 163.5 (C), 165.9 (C). HRMS (ESI) m/z Calculated: $370.0722[\mathrm{M}+\mathrm{H}]^{+}$, Found: $370.0717[\mathrm{M}+\mathrm{H}]^{+}$.

4-\{[( 5- [2-(1H-benzo[d]imidazol-1-yl)ethyl]-1,3,4-thiadiazole-2-yl\}thio)methyl] benzonitrile (8d).

Prepared from 5-(2-(1H-benzo[d]imidazol-1-yl) ethyl)-1,3,4-thiadiazole-2-thiol (7) $(0.1 \mathrm{~g}, 0.38 \mathrm{mmol})$ 
and 4-(chloromethyl)benzonitrile (0.07 g, $0.42 \mathrm{mmol}$ ). Purified using gradient chromatography eluting with $100 \% \mathrm{EtOAc}+1 \% \mathrm{Et}_{3} \mathrm{~N}$ to afford the product as a white solid: Yield: $0.17 \mathrm{gm}, 61 \%$; $\mathrm{mp} 112-114{ }^{\circ} \mathrm{C}$; TLC: $\mathrm{CH}_{2} \mathrm{Cl}_{2}-\mathrm{MeOH}$ 9:1 v/v, $\mathrm{R}_{\mathrm{f}}$ 0.56; HPLC: $100 \%$ at R.T. $4.02 \mathrm{~min} .{ }^{1} \mathrm{H}$ NMR (DMSO- $\left.d_{6}\right) \delta: 3.43(\mathrm{t}, J=6.8 \mathrm{~Hz}$, $\left.2 \mathrm{H}, \mathrm{NCH}_{2} \mathrm{C}_{2}\right), 4.48\left(\mathrm{~s}, 2 \mathrm{H}, \mathrm{SCH}_{2}\right), 4.66(\mathrm{t}, J=6.7 \mathrm{~Hz}$, $\left.2 \mathrm{H}, \mathrm{NCH}_{2}\right), 7.21(\mathrm{~m}, 2 \mathrm{H}, \mathrm{Ar}), 7.57(\mathrm{~m}, 3 \mathrm{H}, \mathrm{Ar}), 7.64(\mathrm{~m}$, $1 \mathrm{H}, \mathrm{Ar}$ ), 7.77 (d, $J=8.4 \mathrm{~Hz}, 2 \mathrm{H}, \mathrm{Ar}), 8.19$ (s, $1 \mathrm{H}$, Imidazole). ${ }^{13} \mathrm{C}$ NMR (DMSO- $\left.d_{6}\right) \delta: 26.2\left(\mathrm{NCH}_{2} \mathrm{CH}_{2}\right), 35.5$ $\left(\mathrm{SCH}_{2}\right), 41.2\left(\mathrm{NCH}_{2}\right), 110.6(\mathrm{CH}), 110.9(\mathrm{CN}), 119.1(\mathrm{C})$, $120.0(\mathrm{CH}), 122.1(\mathrm{CH}), 122.9(\mathrm{CH}), 130.4(2 \mathrm{xCH}), 132.9$ $(2 \times C H), 133.9(\mathrm{C}), 143.2,(\mathrm{C}), 143.8(\mathrm{C}), 144.6(\mathrm{CH}$-imidazole), 163.2 (C), 166.1 (C). HRMS (ESI) $\mathrm{m} / z$ Calculated: $377.0769[\mathrm{M}+\mathrm{H}]^{+}$, Found: $377.0752[\mathrm{M}+\mathrm{H}]^{+}$. 2$\{[2-(1$ H-benzo[d]imidazol-1-yl)ethyl]-

\section{5-(phenethylthio)\}-1,3,4-thiadiazole (9a).}

Prepared from 5-(2-(1H-benzo[d]imidazol-1-yl) ethyl)-1,3,4-thiadiazole-2-thiol (7) (0.1 g, $0.38 \mathrm{mmol})$ and 2-bromoethyl benzene ( $0.07 \mathrm{~g}, 0.42 \mathrm{mmol})$. Purified using gradient chromatography eluting with $100 \%$ EtOAc to afford the product as a white solid: Yield: $0.071 \mathrm{~g}$ (51\%); mp 76-78 ${ }^{\circ} \mathrm{C}$; TLC: $\mathrm{CH}_{2} \mathrm{Cl}_{2}-\mathrm{MeOH} 9: 1 \mathrm{v} / \mathrm{v}, \mathrm{R}_{\mathrm{f}}$ 0.57; HPLC: $100 \%$ at R.T. 4.35 min. ${ }^{1} \mathrm{H}$ NMR (DMSO- $d_{6}$ ) $\delta: 2.97\left(\mathrm{t}, J=7.3 \mathrm{~Hz}, 2 \mathrm{H}, \mathrm{SCH}_{2} \mathrm{C}_{2}\right), 3.39$ (t, $J=7.2 \mathrm{~Hz}$, $\left.2 \mathrm{H}, \mathrm{NCH}_{2} \underline{\mathrm{C}}_{2}\right), 3.44\left(\mathrm{t}, J=6.7 \mathrm{~Hz}, 2 \mathrm{H}, \mathrm{SCH}_{2}\right), 4.69(\mathrm{t}$, $\left.J=6.7 \mathrm{~Hz}, 2 \mathrm{H}, \mathrm{NCH}_{2}\right), 7.19-7.31(\mathrm{~m}, 7 \mathrm{H}, \mathrm{Ar}), 7.59$ (d, $J=7.8 \mathrm{~Hz}, 1 \mathrm{H}, \mathrm{Ar}), 7.63(\mathrm{~d}, J=8.0 \mathrm{~Hz}, 1 \mathrm{H}, \mathrm{Ar}), 8.22(\mathrm{~s}$, $1 \mathrm{H}$, imidazole). ${ }^{13} \mathrm{CNMR}$ (DMSO- $\left.d_{6}\right) \delta: 26.2\left(\mathrm{NCH}_{2} \mathrm{CH}_{2}\right)$, $33.5\left(\mathrm{SCH}_{2}\right), 35.3\left(\mathrm{SCH}_{2} \mathrm{CH}_{2}\right), 41.3\left(\mathrm{NCH}_{2}\right), 110.7(\mathrm{CH})$, $120.0(\mathrm{CH}), 122.1(\mathrm{CH}), 122.9(\mathrm{CH}), 127.0(\mathrm{CH}), 128.9$ $(2 \times C H), 129.1(2 \times C H), 134.0(\mathrm{C}), 139.6(\mathrm{C}), 143.8(\mathrm{C})$, 144.6 (CH-imidazole), 163.9 (C), 165.7 (C). HRMS (ESI) $\mathrm{m} / \mathrm{z}$ Calculated: $366.0973[\mathrm{M}+\mathrm{H}]^{+}$, Found: 366.0962 $[\mathrm{M}+\mathrm{H}]^{+}$.

2- \{[2-(1H-benzo[d]imidazol-1-yl)ethyl]-5- [(4-chlorophenethyl)thio]\}-1,3,4-thiadiazole (9b).

Prepared from 5-(2-(1H-benzo[d]imidazol-1-yl) ethyl)-1,3,4-thiadiazole-2-thiol (7) $(0.1 \mathrm{~g}, 0.38 \mathrm{mmol})$ and 4-chlorophenethyl bromide $(0.092 \mathrm{~g}, 0.42 \mathrm{mmol})$. Purified using gradient chromatography eluting with $100 \%$ EtOAc to afford the product as a white solid: Yield: $0.06 \mathrm{~g}$ (40\%); mp 96-98 ${ }^{\circ} \mathrm{C}$; TLC: $\mathrm{CH}_{2} \mathrm{Cl}_{2}-\mathrm{MeOH}$ 9:1 v/v, $\mathrm{R}_{\mathrm{f}}$ 0.60; HPLC: $100 \%$ at R.T. 4.49 min. ${ }^{1} \mathrm{H}$ NMR (DMSO- $d_{6}$ ) $\delta: 2.96\left(\mathrm{t}, J=7.2 \mathrm{~Hz}, 2 \mathrm{H}, \mathrm{SCH}_{2} \mathrm{CH}_{2}\right), 3.38(\mathrm{t}, J=7.1 \mathrm{~Hz}$, $\left.2 \mathrm{H}, \mathrm{NCH}_{2} \mathrm{C}_{2}\right), 3.44\left(\mathrm{t}, J=6.8 \mathrm{~Hz}, 2 \mathrm{H}, \mathrm{SCH}_{2}\right), 4.69(\mathrm{t}$, $\left.J=6.7 \mathrm{~Hz}, 2 \mathrm{H}, \mathrm{NCH}_{2}\right), 7.20(\mathrm{~m}, 1 \mathrm{H}, \mathrm{Ar}), 7.25(\mathrm{~m}, 3 \mathrm{H}, \mathrm{Ar})$, 7.35 (d, $J=8.5 \mathrm{~Hz}, 2 \mathrm{H}, \mathrm{Ar}), 7.59$ (d, $J=7.7 \mathrm{~Hz}, 1 \mathrm{H}, \mathrm{Ar})$, 7.63 (d, J=7.8 Hz, 1H, Ar), 8.22 (s, $1 \mathrm{H}$, imidazole). ${ }^{13} \mathrm{C}$ NMR (DMSO- $\left.d_{6}\right) \delta: 26.2\left(\mathrm{NCH}_{2} \underline{\mathrm{CH}_{2}}\right), 33.3\left(\mathrm{SCH}_{2}\right), 34.5$ $\left(\mathrm{SCH}_{2} \mathrm{CH}_{2}\right), 41.3\left(\mathrm{NCH}_{2}\right), 110.7(\mathrm{CH}), 120.0(\mathrm{CH}), 122.1$ $(\mathrm{CH}), 122.9(\mathrm{CH}), 128.8(2 \times \mathrm{CH}), 131.0(2 \times \mathrm{CH}), 131.7(\mathrm{C})$, 134.0 (C), 138.6 (C), 143.8 (C), 144.6 (CH-imidazole),
163.8 (C), 165.8 (C). HRMS (ESI) $m / z$ Calculated: $400.0583[\mathrm{M}+\mathrm{H}]^{+}$, Found: $400.0555[\mathrm{M}+\mathrm{H}]^{+}$.

2- \{[2-(1H-benzo[d]imidazol-1-yl)ethyl]-5- [(4-methoxyphenethyl)thio]\}-1,3,4-thiadiazole (9c).

Prepared from 5-(2-(1H-benzo[d]imidazol-1-yl) ethyl)-1,3,4-thiadiazole-2-thiol (7) (0.1 g, $0.38 \mathrm{mmol})$ and 4-methoxyphenethyl bromide $(0.09 \mathrm{~g}, 0.42 \mathrm{mmol})$. Purified using gradient chromatography eluting with $100 \%$ EtOAc to afford the product as a white solid: Yield: $0.166 \mathrm{~g}$ (55\%); mp $68-70{ }^{\circ} \mathrm{C}$; TLC: $\mathrm{CH}_{2} \mathrm{Cl}_{2}-\mathrm{MeOH}$ 9:1 $\mathrm{v} / \mathrm{v}, \mathrm{R}_{\mathrm{f}} 0.66$; HPLC: $100 \%$ at $\mathrm{RT}=4.34 \mathrm{~min} .{ }^{1} \mathrm{H}$ NMR (DMSO- $d_{6}$ ) $\delta: 2.89$ (t, $J=7.1 \mathrm{~Hz}, 2 \mathrm{H}, \mathrm{SCH}_{2} \mathrm{CH}_{2}$ ), $\mathrm{CH}_{2}$ signal is obscured by $\mathrm{H}_{2} \mathrm{O}$ in DMSO- $d_{6}$ signal, 3.44 $\left(\mathrm{t}, J=6.7 \mathrm{~Hz}, 2 \mathrm{H}, \mathrm{SCH}_{2}\right), 3.73\left(\mathrm{~s}, 3 \mathrm{H}, \mathrm{OCH}_{3}\right), 4.69(\mathrm{t}$, $\left.J=6.7 \mathrm{~Hz}, 2 \mathrm{H}, \mathrm{NCH}_{2}\right), 6.85(\mathrm{~d}, J=8.7 \mathrm{~Hz}, 2 \mathrm{H}, \mathrm{Ar}), 7.13$ (d, $J=8.7 \mathrm{~Hz}, 2 \mathrm{H}, \mathrm{Ar}), 7.20$ (td, $J=1.2,7.2 \mathrm{~Hz}, 1 \mathrm{H}, \mathrm{Ar})$, $7.25(\mathrm{td}, J=1.2,8.1 \mathrm{~Hz}, 1 \mathrm{H}, \mathrm{Ar}), 7.59(\mathrm{~d}, J=7.8 \mathrm{~Hz}, 1 \mathrm{H}$, Ar), 7.63 (d, $J=7.8 \mathrm{~Hz}, 1 \mathrm{H}, \mathrm{Ar}), 8.22$ (s, $1 \mathrm{H}$, imidazole).

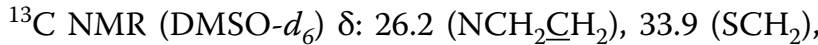
$34.4\left(\mathrm{SCH}_{2} \mathrm{CH}_{2}\right), 41.3\left(\mathrm{NCH}_{2}\right), 55.5\left(\mathrm{OCH}_{3}\right), 110.7(\mathrm{CH})$, $114.3(2 \times C H), 120.0(\mathrm{CH}), 122.1(\mathrm{CH}), 122.9(\mathrm{CH}), 130.1$ $(2 \times C H), 131.5(\mathrm{C}), 140.0(\mathrm{C}), 143.8(\mathrm{C}), 144.6(\mathrm{CH}$-imidazole), 158.4 (C), 164.0 (C), 165.7 (C). HRMS (ESI): Calculated: $380.1307[\mathrm{M}+\mathrm{H}]^{+}$, Found: $380.1319[\mathrm{M}+\mathrm{H}]^{+}$.

\section{Computational studies}

Flexible alignment studies were performed using MOE. 2015.10 software [31]. Flexible alignment was performed using MMFF94 forcefield, flexible alignment mode and the resulting conformations were examined according to their grand alignment score (S). The latter is the sum of the similarity measure of configuration (F) and the average strain energy of the molecules in the alignment in $\mathrm{kcal} / \mathrm{mol}(\mathrm{U})$. The lower $\mathrm{S}$ value indicates better alignment. Multiple sequence alignment was performed using the EMBL-EBI (European Bioinformatics Institute) Job Dispatcher framework server, using Clustal Omega [33], while the amino acid sequences were imported in their FASTA formats from UniProtKB [47].

Docking studies were performed using MOE. 2015.10 software [48] using the crystal structure of Th. thermophilus PheRS [PDB ID: 1JJC] [32]. All minimisations were performed with MOE until a RMSD gradient of $0.01 \mathrm{kcal} / \mathrm{mol} / \AA$ with Amber99 forcefield and automatic calculation of the partial charges. Docking settings were set as the following: Amber99 forcefield for result refinement, Triangle Matcher placement was chosen to determine the poses, London $\Delta G$ scoring function was applied for rescoring and result refinement, and the structures were further refined with rigid receptor refinement. The resulting docking poses were generated in a database, 
which was arranged according to the final score function (S) that is the score of the last stage which was not set to zero.

Molecular dynamic simulations were run using either the crystal structure of Th. thermophilus PheRS [PDB ID: 1JJC] [32] or S. aureus PheRS previously published homology model [34]. PDB files were first optimised using protein preparation wizard in Maestro [49] version 11.8.012 by assigning bond orders, adding hydrogen, and correcting incorrect bond types. A default quick relaxation protocol was used to minimise the MD systems with the Desmond programme [49]. In Desmond, the volume of space in which the simulation takes place, the global cell, is built up by regular 3D simulation boxes, which was utilised as part of this system for protein interactions. The simulation system was generated by embedding the protein model in an orthorhombic $10 \AA$ water box. Overlapping water molecules were deleted. The solvated system was neutralised by adding sodium ions and salt concentration $0.15 \mathrm{M}$. Force-field parameters for the complexes were assigned using the OPLS_2005 forcefield, that is, a $200 \mathrm{~ns}$ molecular dynamic run in the NPT ensemble $(\mathrm{T}=300 \mathrm{~K})$ at a constant pressure of $1 \mathrm{bar}$. Energy and trajectory atomic coordinate data were recorded at each $1.2 \mathrm{~ns}$.

Binding affinity $(\Delta G)$ calculations was performed using Prime/MMGBAS, available in the Schrödinger Prime suite, to calculate the binding free energy of $\mathbf{8 b}$ complexed with the $S$. aureus homology model.

$\Delta \mathrm{G}$ (bind) $=\mathrm{E} \_$complex (minimised) $-\left(\mathrm{E} \_\right.$ligand (minimised) + E_receptor (minimised)) .

The mean $\Delta G$ (bind) was calculated from each frame starting from the last $100 \mathrm{~ns}$ to the final frame of the MD simulation.

\section{Microbiological evaluation}

Compounds 5a-d, 6a-c, 8a-d and 9a-c were evaluated using the guidelines of ISO20776 (the International Organization of Standardization) broth microdilution method using Muller-Hinton broth and concentration range of $0.008-128 \mu \mathrm{g} / \mathrm{mL}$ [50]. The MIC values were determined against $S$. aureus (ATCC 29,213), E. faecalis (ATCC 29,212), P. aeruginosa (ATCC 29,853), E. coli (ATCC 25,922) and Klebsiella pneumoniae (ATCC $700,603)$ using ciprofloxacin as a reference drug. The tests were performed in microdilution trays and the amount of growth in each well was compared with that in the positive growth control, and the recorded MIC is the lowest concentration of the agent that completely inhibits visible growth.

\section{Abbreviations}

PheRS: Phenylalanyl-tRNA synthetase; aa-AMP: Aminoacyl adenylate; Phe-AMP: Phenylalanyl adenylate; MD: Molecular dynamics; MIC: Minimum inhibitory concentration; aaRSs: Aminoacyl tRNA synthetases; PheS: Phenylalanyl tRNA synthetase a subunit; PheT: Phenylalanyl tRNA synthetase $\beta$ subunit; ATP: Adenosine triphosphate; PDB: Protein data bank; DMF: Dimethylformamide; MOE: Molecular operating environment.

\section{Supplementary Information}

The online version contains supplementary material available at https://doi. org/10.1186/s13065-021-00785-8.

Additional file 1. Contains: a- Fig S1. Figure illustrating the ${ }^{1} \mathrm{H}$ NMR spectrum of compound $6 \mathrm{~b}, \mathrm{~b}$ - The ${ }^{1} \mathrm{H}$ NMR and ${ }^{13} \mathrm{C}$ NMR charts of the target compounds and c-The HPLC traces of the target compounds.

\section{Acknowledgements}

We thank the Egyptian Ministry of Higher Education-Mission Sector, and the British Council for funding this research in Cardiff University through a Newton-Mosharafa scholarship to Nada A. Noureldin. Molecular dynamics simulations were undertaken using the supercomputing facilities at Cardiff University operated by Advanced Research Computing at Cardiff (ARCCA) on behalf of the Cardiff Supercomputing Facility and the HPC Wales and Supercomputing Wales (SCW) projects. We acknowledge support of the latter, which is part-funded by the European Regional Development Fund (ERDF) via the Welsh Government.

\section{Authors' contributions}

CS designed the research work, supervised the practical experiments, performed the MD simulations, and revised the manuscript, NA synthesised and purified the compounds, carried out the ${ }^{1} \mathrm{H} N M R,{ }^{13} \mathrm{C}$ NMR, performed the rest of the computational work and wrote the manuscript. MW supervised the biological tests. JR performed the biological tests. HK revised the manuscript and supervised the research. MB and SM reviewed the manuscript. All authors read and approved the final manuscript.

\section{Funding}

This work was funded by the Egyptian Ministry of Higher Education-Mission Sector, and the British Council, Egypt and performed in Cardiff University through a Newton-Mosharafa scholarship to Nada A. Noureldin.

\section{Availability of data and materials}

The datasets used or analysed during the current study are available from the corresponding author on reasonable request.

\section{Declarations}

\section{Ethics approval and consent to participate}

The manuscript does not contain studies with animal subjects.

\section{Consent for publication}

Not applicable.

\section{Competing interests}

The authors declare that they have no competing interests.

\section{Author details}

${ }^{1}$ School of Pharmacy and Pharmaceutical Sciences, Cardiff University, Cardiff CF10 3NB, UK. ${ }^{2}$ Department of Medicinal Chemistry, Faculty of Pharmacy, Zagazig University, Zagazig P.C. 44519, Egypt. ${ }^{3}$ Specialist Antimicrobial Chemotherapy Unit, University Hospital of Wales, Heath Park, Cardiff CF14 4XW, UK.

Received: 17 August 2021 Accepted: 20 October 2021

Published online: 28 October 2021 


\section{References}

1. Watkins RR, Bonomo RA. Overview: the ongoing threat of antimicrobial resistance. Infect Dis Clin North Am. 2020;34:649-58.

2. Morrison L, Zembower TR. Antimicrobial resistance. Gastrointest Endosc Clin N Am. 2020;30:619-35.

3. Ragheb MN, Thomason MK, Hsu C, Nugent P, Gage J, Samadpour AN, Kariisa A, Merrikh CN, Miller SI, Sherman DR. Inhibiting the evolution of antibiotic resistance. Mol Cell. 2019;73:157-65.

4. Cassini A, Högberg LD, Plachouras D, Quattrocchi A, Hoxha A, Simonsen GS, Colomb-Cotinat M, Kretzschmar ME, Devleesschauwer B, Cecchini M. Attributable deaths and disability-adjusted life-years caused by infections with antibiotic-resistant bacteria in the EU and the European Economic Area in 2015: a population-level modelling analysis. Lancet Infect Dis. 2019;19:56-66.

5. Jenkins DR. Nosocomial infections and infection control. Medicine. 2017:45:629-33

6. Abushaheen MA, Fatani AJ, Alosaimi M, Mansy W, George M, Acharya S, Rathod S, Divakar DD, Jhugroo C, et al. Antimicrobial resistance, mechanisms and its clinical significance. Dis Mon. 2020;66:100971.

7. Munita JM, Arias CA. Mechanisms of antibiotic resistance. Virulence mechanisms of bacterial pathogens. ASM Press; 2016. p. 481-511.

8. Ibba M, Söll D. Aminoacyl-tRNA synthesis. Annu Rev Biochem. 2000;69:617-50

9. Hurdle JG, O'Neill AJ, Chopra I. Prospects for aminoacyl-tRNA synthetase inhibitors as new antimicrobial agents. Antimicrob Agents Chemother. 2005; 49:4821-33.

10. Torres-Larios A, Dock-Bregeon A-C, Romby P, Rees B, Sankaranarayanan R, Caillet J, Springer M, Ehresmann C, Ehresmann B, Moras D. Structural basis of translational control by Escherichia coli threonyl tRNA synthetase. Nat Struct Biol. 2002;9:343-7.

11. Arnez J, Harris D, Mitschler A, Rees B, Francklyn C, Moras D. Crystal structure of histidyl-tRNA synthetase from Escherichia coli complexed with histidyl-adenylate. EMBO J. 1995;14:4143-55.

12. Yaremchuk A, Tukalo M, Grøtli M, Cusack S. A succession of substrate induced conformational changes ensures the amino acid specificity of Thermus thermophilus prolyl-tRNA synthetase: comparison with histidyltRNA synthetase. J Mol Biol. 2001;309:989-1002.

13. Arnez JG, Dock-Bregeon A-C, Moras D. Glycyl-tRNA synthetase uses a negatively charged pit for specific recognition and activation of glycine. J Mol Biol. 1999;286:1449-59.

14. Rees B, Webster G, Delarue M, Boeglin M, Moras D. Aspartyl tRNA-synthetase from Escherichia coli: flexibility and adaptability to the substrates. J Mol Biol. 2000;299:1157-64.

15. Sekine SI, Nureki O, Dubois DY, Bernier S, Chênevert R, Lapointe J, Vassylyev DG, Yokoyama S. ATP binding by glutamyl-tRNA synthetase is switched to the productive mode by tRNA binding. EMBO J. 2003;22:676-88

16. Belrhali $H$, Yaremchuk $A$, Tukalo M, Larsen K, Berthet-Colominas C, Leberman R, Beijer B, Sproat B, Als-Nielsen J, Grubel G. Crystal structures at 2.5 angstrom resolution of seryl-tRNA synthetase complexed with two analogs of seryl adenylate. Science. 1994;263:1432-6.

17. Gomez MAR, Ibba M. Aminoacyl-tRNA synthetases. RNA. 2020;26:910-36.

18. Rajendran $V$, Kalita P, Shukla H, Kumar A, Tripathi T. Aminoacyl-tRNA synthetases: structure, function, and drug discovery. Int J Biol Macromol. 2018;111:400-14.

19. Vondenhoff $\mathrm{GH}$, Van Aerschot A. Aminoacyl-tRNA synthetase inhibitors as potential antibiotics. Eur J Med Chem. 2011:46:5227-36.

20. Kwon NH, Fox PL, Kim S. Aminoacyl-tRNA synthetases as therapeutic targets. Nat Rev Drug Discovery. 2019;18:629-50.

21. Pang YLJ, Poruri $K$, Martinis SA. tRNA synthetase: tRNA aminoacylation and beyond. Wiley Interdiscip Rev: RNA. 2014;5:461-80.

22. Fang P, Guo M. Evolutionary limitation and opportunities for developing tRNA synthetase inhibitors with 5-binding-mode classification. Life. 2015;5:1703-25.

23. Chakraborty S, Banerjee R. Phenylalanyl-tRNA synthetase. Res Rep Biochem. 2016:6:25-38.

24. Mermershtain I, Finarov I, Klipcan L, Kessler N, Rozenberg H, Safro MG. Idiosyncrasy and identity in the prokaryotic Phe-system: crystal structure of E. coli phenylalanyl-tRNA synthetase complexed with phenylalanine and AMP. Protein Sci. 2011;20:160-7.
25. Reshetnikova L, Moor N, Lavrik O, Vassylyev DG. Crystal structures of phenylalanyl-tRNA synthetase complexed with phenylalanine and a phenylalanyl-adenylate analogue. J Mol Biol. 1999;287:555-68.

26. Goldgur Y, Mosyak L, Reshetnikova L, Ankilova V, Lavrik O, Khodyreva S, Safro M. The crystal structure of phenylalanyl-tRNA synthetase from Thermus thermophilus complexed with cognate tRNA ${ }^{\text {Phe }}$. Structure. 1997:5:59-68.

27. Evdokimov AG, Mekel M, Hutchings K, Narasimhan L, Holler T, McGrath T, Beattie B, Fauman E, Yan C, Heaslet H. Rational protein engineering in action: the first crystal structure of a phenylalanine tRNA synthetase from Staphylococcus haemolyticus. J Struct Biol. 2008;162:152-69.

28. Abibi A, Ferguson AD, Fleming PR, Gao N, Hajec LI, Hu J, Laganas VA, McKinney DC, McLeod SM, Prince DB. The role of a novel auxiliary pocket in bacterial phenylalanyl-tRNA synthetase druggability. J Biol Chem. 2014;289:21651-62.

29. Wang H, Xu M, Engelhart CA, Zhang X, Yan B, Pan M, Xu Y, Fan S, Liu R, Xu L. Re-discovery of PF-3845 as a new chemical scaffold inhibiting phenylalanyl-tRNA synthetase in Mycobacterium tuberculosis. J Biol Chem. 2021;296:100257.

30. Baidin V, Owens TW, Lazarus MB, Kahne D. Simple secondary amines inhibit growth of gram-negative bacteria through highly selective binding to phenylalanyl-tRNA synthetase. J Am Chem Soc. 2021;143:623-7.

31. Labute P, Williams C, Feher M, Sourial E, Schmidt JM. Flexible alignment of small molecules. J Med Chem. 2001;44:1483-90.

32. Fishman R, Ankilova V, Moor N, Safro M. Structure at $2.6 \AA$ resolution of phenylalanyl-tRNA synthetase complexed with phenylalanyl-adenylate in the presence of manganese. Acta Cryst D. 2001;57:1534-44.

33. Madeira F, Park YM, Lee J, Buso N, GurT, Madhusoodanan N, Basutkar P, Tivey ARN, Potter SC, Finn RD et al. The EMBL-EBI search and sequence analysis tools APIs in 2019. Nucleic Acids Res. 2019; 47:W636-W641. https://www.ebi.ac.uk/Tools/msa/clustalo/.

34. Elbaramawi SS, Ibrahim SM, Lashine ESM, El-Sadek ME, Mantzourani E. Simons C (2017) Exploring the binding sites of Staphylococcus aureus phenylalanine tRNA synthetase: a homology model approach. J Mol Graph Model. 2017;73:36-47.

35. Eissa AG, Blaxland JA, Williams RO, Metwally KA, El-Adl SM, Lashine E-SM, Baillie LW, Simons C. Targeting methionyl tRNA synthetase: design, synthesis and antibacterial activity against Clostridium difficile of novel 3-biaryl-N-benzylpropan-1-amine derivatives. J Enzyme Inhib Med Chem. 2016:31:1694-7.

36. Eissa AG, Blaxland JA, Williams RO, Metwally KA, El-Adl SM, Lashine E-SM, Baillie LWJ, Simons C. Adenine and benzimidazole-based mimics of REP-3123 as antibacterial agents against Clostridium difficile and Bacillus anthracis: design, synthesis and biological evaluation. Bull Fac Pharm Cairo Univ. 2016;54:197-207.

37. Bhanushali MJ, Nandurkar NS, Jagtap SR, Bhanage BM. Y $\left(\mathrm{NO}_{3}\right)_{3} \cdot 6 \mathrm{H}_{2} \mathrm{O}$ catalyzed aza-Michael addition of aromatic/hetero-aromatic amines under solvent-free conditions. Catal Commun. 2008;9:1189-95.

38. Poritere SE, Paégle RA, Lidak MY. Synthesis of modified amino acids containing nucleic acid purine bases. Chem Heterocycl Compd. 1985:21:104-7.

39. Elbaramawi SS, Hughes C, Richards J, Gupta A, Ibrahim SM, Lashine E-SM, El-Sadek ME, O'Neill AJ, Wootton M, Bullard JM, Simons C. Design, synthesis and microbiological evaluation of novel compounds as potential Staphylococcus aureus phenylalanine tRNA synthetase inhibitors. Egypt J Chem. 2018;61:9-25.

40. Sauer AC, Leal JG, Stefanello ST, Leite MT, Souza MB, Soares FA, Rodrigues OE, Dornelles L. Synthesis and antioxidant properties of organosulfur and organoselenium compounds derived from 5-substituted-1, 3, 4-oxadiazole/thiadiazole-2-thiols. Tetrahedron Lett. 2017:58:87-91.

41. Beyer D, Kroll H-P, Endermann R, Schiffer G, Siegel S, Bauser M, Pohlmann J, Brands M, Ziegelbauer K, Haebich D. New class of bacterial phenylalanyl-tRNA synthetase inhibitors with high potency and broad-spectrum activity. Antimicrob Agents Chemother. 2004;48:525-32.

42. Xiang YY, Finn J, Hill JM, Wang ZG, Keith D, Silverman J, Oliver N. A series of spirocyclic analogues as potent inhibitors of bacterial phenylalanyltRNA synthetases. Bioorg Med Chem Lett. 2004;14:1339-42.

43. Jarvest RL, Erskine SG, Forrest AK, Fosberry AP, Hibbs MJ, Jones JJ, O'Hanlon PJ, Sheppard RJ, Worby A. Discovery and optimisation of potent, selective, ethanolamine inhibitors of bacterial phenylalanyl tRNA synthetase. Bioorg Med Chem Lett. 2005;15:2305-9. 
44. Montgomery Jl, Toogood PL, Hutchings KM, Liu J, Narasimhan L, Braden T, Dermyer MR, Kulynych AD, Smith YD, Warmus JS. Discovery and SAR of benzyl phenyl ethers as inhibitors of bacterial phenylalanyl-tRNA synthetase. Bioorg Med Chem Lett. 2009;19:665-9.

45. Wang L-N, Di W-J, Zhang Z-M, Zhao L-L, Zhang T, Deng Y-R, Yu L-Y. Small-molecule inhibitors of the tuberculosis target, phenylalanyl-tRNA synthetase from Penicillium griseofulvum CPCC-400528. Cogent Chem. 2016;2:1181536

46. Demirayak \$, Benkli K, Güven K. Synthesis and antimicrobial activities of some 3-arylamino-5- [2-(substituted 1-imidazolyl)ethyl]-1, 2, 4-triazole derivatives. Eur J Med Chem. 2011;35:1037-40.

47. Consortium TU. UniProt: a worldwide hub of protein knowledge. Nucleic Acids Res. 2018; 47:D506-D515. https://www.uniprot.org/.

48. Molecular Operating Environment (MOE). Chemical Computing Group ULC; 2019. https://www.chemcomp.com/Products.htm

49. Schrödinger Release 2021-2: Desmond Molecular Dynamics System, D. E. Shaw Research. New York, NY, 2021. Maestro-Desmond Interoperability
Tools, Schrödinger, New York, NY, 2021. https://www.schrodinger.com/ products/desmond.

50. European Committee on Antimicrobial Susceptibility Testing. ISO 20776-1: 2019: Susceptibility testing of infectious agents and evaluation of performance of antimicrobial susceptibility test devices Part 1 : Broth microdilution reference method for testing the in vitro activity of antimicrobial agents against rapidly growing aerobic bacteria involved in infectious diseases. International Organization for Standardization. https://www.iso.org/standard/70464.html.2019].

\section{Publisher's Note}

Springer Nature remains neutral with regard to jurisdictional claims in published maps and institutional affiliations.
Ready to submit your research? Choose BMC and benefit from:

- fast, convenient online submission

- thorough peer review by experienced researchers in your field

- rapid publication on acceptance

- support for research data, including large and complex data types

- gold Open Access which fosters wider collaboration and increased citations

- maximum visibility for your research: over 100M website views per year

At BMC, research is always in progress.

Learn more biomedcentral.com/submissions 